\title{
Synthesis, Structure and Electronic Properties of Graphitic Carbon Nitride Films
}

Theo Suter, ${ }^{*}, \dagger, \nabla$ Veronika Brázdová, ${ }^{\dagger}$ Kit McColl, ${ }^{\dagger}$ Thomas S. Miller, $^{\dagger, \nabla}$ Hiroki Nagashima, ${ }^{\S, \perp, q[}$ Enrico Salvadori, ${ }^{\S, \|}$ Andrea Sella, ${ }^{\dagger}$ Christopher A. Howard, ${ }^{\ddagger}{ }^{\ddagger}$ Christopher W. M. Kay, ${ }^{\S, \#}$ Furio Corà, ${ }^{\dagger}$ and Paul F. McMillan* ${ }^{*} \dagger$

${ }^{\dagger}$ Department of Chemistry, University College London, Christopher Ingold Building, 20 Gordon Street, London WC1H 0AJ, U.K.

"Department of Physics and Astronomy, University College London, Gower Street, London WC1E 6BT, U.K.

${ }^{\S}$ Institute of Structural and Molecular Biology and London Centre for Nanotechnology, University College London, London WE1E 6BT, U.K.

"School of Biological and Chemical Sciences, Queen Mary University of London, London E1 4NS, U.K.

${ }^{\perp}$ Division of Material Science, Graduate School of Science, Nagoya University, Furo-cho, Chikusa, Nagoya 464-8602, Japan

\#Department of Chemistry, University of Saarland, 66123 Saarbrücken, Germany

"Molecular Photoscience Research Center, Kobe University, 1-1 Rokkodai-cho, Nada-ku, Kobe 657-8501, Japan

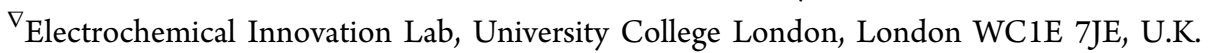

Supporting Information

ABSTRACT: Dark-colored shiny flakes of graphitic carbon nitride materials produced by reacting dicyandiamide $\mathrm{C}_{2} \mathrm{~N}_{4} \mathrm{H}_{4}$ in a $\mathrm{KBr} / \mathrm{LiBr}$ molten salt medium were determined to have a $\mathrm{C} / \mathrm{N}$ ratio near 1.2:1. The compounds also contained 2.3-2.5 wt \% $\mathrm{H}$ incorporated within $\mathrm{N}-\mathrm{H}$ species identified by Fourier transform infrared spectroscopy. One recent study revealed analogous results for thin films produced by an similar synthesis method, while a previous investigation instead reported formation of crystalline $\mathrm{gC}_{3} \mathrm{~N}_{4}$ flakes with a triazine-based graphitic carbon nitride (TGCN) structure. The structures of the materials produced here were studied
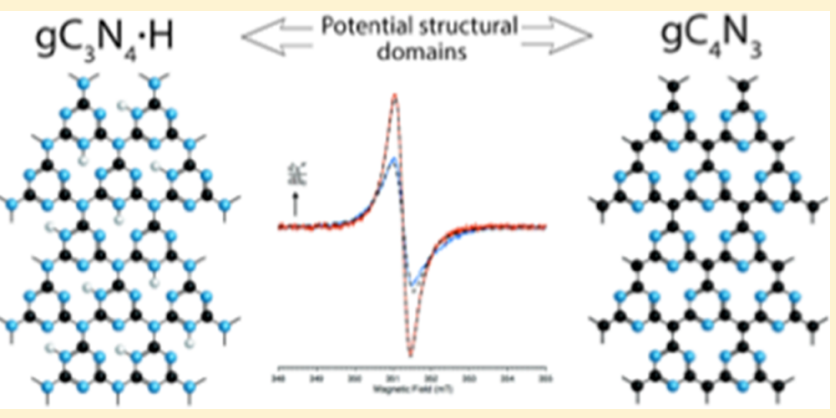
using a combination of high resolution transmission electron microscopy, X-ray diffraction, IR and Raman and X-ray photoelectron spectroscopy, along with series of density functional theory (DFT) calculations carried out for a range of model layered structures. The results indicate the graphitic layered $\mathrm{gC}_{x} \mathrm{~N}_{y}$ materials contain a mixture of $\mathrm{sp}^{2}$-hybridized $\mathrm{C}-\mathrm{N}$ and $\mathrm{C}-\mathrm{C}$ bonded structures, with TGCN to graphene-like domains existing within the layers. Paramagnetic centers localized on the $\mathrm{C}_{3} \mathrm{~N}_{3}$ rings revealed by electron paramagnetic resonance spectroscopy correspond to potential defect structures within the graphitic layers predicted by DFT calculations. Our results combined with those of previous researchers indicate that a range of graphitic carbon nitride materials could exist with different $\mathrm{C} / \mathrm{N} / \mathrm{H}$ ratios leading to tunable electronic properties for catalysis, semiconducting, spintronics and energy applications, that could be targeted by controlling the synthesis and thin film deposition procedures.

\section{INTRODUCTION}

Layered carbon-based materials are being developed for applications ranging from optoelectronics, energy storage and conversion to catalysis and photocatalysis. ${ }^{1-18}$ Graphitic carbon nitride $\left(\mathrm{gC}_{x} \mathrm{~N}_{y}\right)$ materials are typically reported to be wide-gap semiconductors, with bandgaps ranging between 2.4 and $2.7 \mathrm{eV}$. Most $\mathrm{C}_{x} \mathrm{~N}_{y} \mathrm{H}_{z}$ compounds currently under investigation correspond to amorphous to nanocrystalline polymers to layered materials formed by thermal condensation of $\mathrm{N}$-rich precursors such as dicyandiamide $\left(\mathrm{C}_{2} \mathrm{~N}_{4} \mathrm{H}_{4}\right.$ : DCDA), melamine or urea by elimination of $\mathrm{NH}_{3}$. Although the end result of such a process is predicted be a fully-polymerized $\mathrm{gC}_{3} \mathrm{~N}_{4}$ phase, this is not achieved due to evaporation of $\mathrm{CN}$-rich components at higher temperatures, and the products typically have a limiting composition near $\mathrm{C}_{2} \mathrm{~N}_{3} \mathrm{H}$. However, synthesis of $\mathrm{gC}_{3} \mathrm{~N}_{4}$ films has been achieved by chemical vapour deposition techniques using single-source precursors. ${ }^{1,2}$ The nanocrystalline materials produced were characterized in that study by high resolution transmission electron microscopy (HRTEM), electron energy loss spectroscopy, Fourier transform infrared (FTIR) and Rutherford backscattering. Fully condensed $\mathrm{gC}_{3} \mathrm{~N}_{4}$ could be based on tri-s-triazine (heptazine, $\left.\mathrm{C}_{6} \mathrm{~N}_{7}\right)$ or s-triazine $\left(\mathrm{C}_{3} \mathrm{~N}_{3}\right)$

Received: August 16, 2018

Revised: October 13, 2018

Published: October 22, 2018 

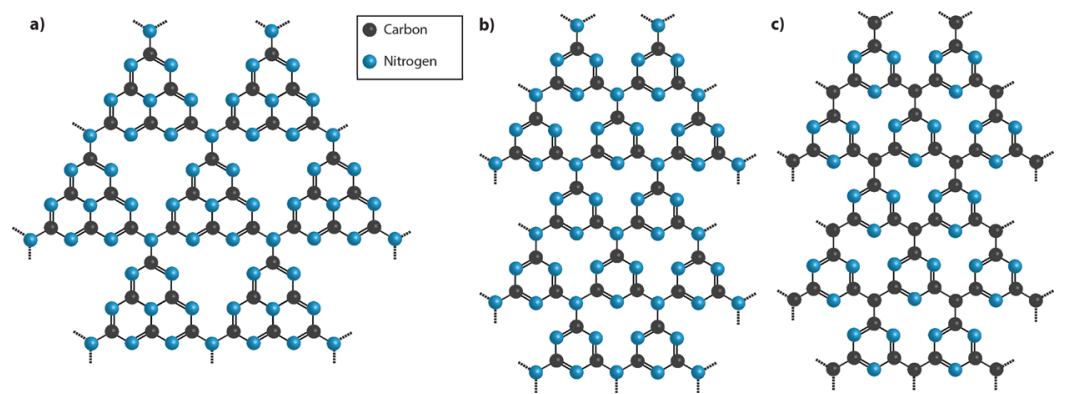

Figure 1. Schematic diagrams of different structural motifs for carbon nitride layered structures. (a) $\mathrm{PH}_{\mathrm{gC}} \mathrm{N}_{4}$ (b) polytriazine gC $\mathrm{C}_{3} \mathrm{~N}_{4}$ (c) polytriazine $\mathrm{gC}_{4} \mathrm{~N}_{3}$.

(Figure 1a,b) units, with polyheptazine $(\mathrm{PH})$ layered structures predicted to give rise to more thermodynamically stable forms. ${ }^{3,4}$ However, such structures have not been observed experimentally. Instead, the materials prepared by Kouvetakis et al. were found to be based on $\mathrm{C}_{3} \mathrm{~N}_{3}$ rings linked by threecoordinated nitrogen $\left(\mathrm{sp}^{2}\right.$-bonded $\left.\mathrm{NC}_{3}\right)$ units, forming graphene-like sheets containing $\mathrm{C}_{6} \mathrm{~N}_{6}$ voids within the layers (Figure $1 \mathrm{~b}$ ). Various $\mathrm{gC}_{3} \mathrm{~N}_{4}$ polymorphs based on different stacking arrangements of both the polytriazine (PT) and $\mathrm{PH}$ layer motifs have been studied theoretically., $3-9$

Other investigations discovered that reacting precursors such as melamine and cyanuric chloride under high pressure-high temperature (HPHT) conditions gave rise to a new class of crystalline carbon nitrides, that were based on polytriazine units linked by bridging imido - $\mathrm{NH}-$ groups to form layered polytriazine imide (PTI) structures with $\mathrm{C}_{12} \mathrm{~N}_{12}$ voids within the graphitic sheets. ${ }^{10,11}$ The PTI $x \mathrm{HCl}$ compounds reported in the HPHT study contained $\mathrm{Cl}^{-}$ions occupying intralayer void sites with additional charge-balancing $\mathrm{H}^{+}$ions attached to $\mathrm{N}$ atoms around the ring. Formation of a related series of crystalline PTI compounds was then reported by reacting DCDA in $\mathrm{LiCl} / \mathrm{KCl}$ or $\mathrm{LiBr} / \mathrm{KBr}$ molten salt media. ${ }^{12-14} \mathrm{Here}^{+}{ }^{+}$partly replaced $\mathrm{H}^{+}$ ions around the $\mathrm{C}_{12} \mathrm{~N}_{12}$ ring voids, and $\mathrm{Cl}^{-}$or $\mathrm{Br}^{-}$ions now occupied interlayer sites.

During these syntheses a dark-colored shiny material is typically deposited on the walls of the reaction chamber, or is found among the main products contained within the salt block derived from the solidified reaction medium. Algara-Siller et al. ${ }^{8}$ addressed characterization of this additional phase, using HRTEM, X-ray diffraction (XRD), spectroscopic and density functional theory (DFT) techniques to study the micrometersized flakes that formed at the surface of the molten salt or were deposited on the walls of the container. ${ }^{8}$ Their analyses indicated that the material was $\mathrm{gC}_{3} \mathrm{~N}_{4}$ with a triazine-based layered structure (TGCN), that then represented the first bulk crystalline example of the nanocrystalline phase found by Kouvetakis et al., ${ }^{1,2}$ and that was included in the ab initio theoretical predictions of Teter and Hemley. ${ }^{5}$ It was proposed that the semiconducting TGCN phase could then be developed for a wide range of optoelectronics, catalysis and solar energy harvesting applications. ${ }^{15,16}$

Other layered phases that have also been described as layered "carbon nitrides" include $\mathrm{N}$-doped graphites and graphenes, that have metallic properties. ${ }^{17-21}$ Nanocomposites containing interleaved $\mathrm{gC}_{3} \mathrm{~N}_{4}$ and graphene sheets have been proposed as multifunctional materials with tunable electronic properties leading to a wide range of potential applications. ${ }^{22}$ Recent work has predicted the existence of a new compound $\mathrm{gC}_{4} \mathrm{~N}_{3}$, containing $\mathrm{C}$ atoms substituted for $\mathrm{sp}^{2}$-bonded $\mathrm{N}$ on sites linking the triazine rings within TGCN layers (Figure 1c). This compound has an unsaturated valency count resulting in interesting half-metallic magnetic properties. ${ }^{22-24}$ Doping such structures with $\mathrm{H}$ or $\mathrm{Li}$ could suppress the metallicity to provide optically transparent materials with photocatalytic properties. $^{23}$

Ladva et al. recently reported carbon nitride thin films deposited on different supports from vapors obtained by reacting DCDA in a eutectic $\mathrm{LiBr} / \mathrm{KBr}$ molten salt medium. ${ }^{34}$ Their chemical analyses indicated a $\mathrm{C} / \mathrm{N}$ ratio $\sim 1.2: 1$, that is similar to the materials produced in our own study, but that is substantially more C-rich than the TGCN compound investigated by Algara-Siller et al. Here we present results of our detailed characterization studies of layered $\mathrm{gC}_{x} \mathrm{~N}_{y} \mathrm{H}_{z}$ nanomaterials produced by condensation reactions from DCDA in a $\mathrm{LiBr} / \mathrm{KBr}$ molten salt medium.

\section{METHODS}

2.1. Synthesis. Solid DCDA $\left(\mathrm{C}_{2} \mathrm{~N}_{4} \mathrm{H}_{4}: 0.83\right.$ g, SigmaAldrich) was ground under argon together with a pre-dried mixture of $\mathrm{KBr}$ (5.99 g, Alfa Aesar) and $\mathrm{LiBr}$ (6.48 g, Aldrich) in proportions corresponding to the eutectic melt composition $\left(T_{\mathrm{m}}=329^{\circ} \mathrm{C}\right)$. The mixture was transferred to a quartz glass ampoule (OD $25 \mathrm{~mm}$, ID $22 \mathrm{~mm}$, length $15 \mathrm{~cm}$ ), evacuated to $10^{-5}$ mbar (Leybold vacuum systems, PT70G compact), then sealed under vacuum. The ampoules were heated to $400{ }^{\circ} \mathrm{C}$ at 40 ${ }^{\circ} \mathrm{C} / \mathrm{min}$, maintained for $4 \mathrm{~h}$ at this temperature, and then raised to $600{ }^{\circ} \mathrm{C}$ for $60 \mathrm{~h}$, using a horizontal tube furnace (Carbolite MTF 12/38/400). Some synthesis experiments were also carried out in a vertical (Deltech) furnace but yields of the dark-colored shiny product to be studied here were found to be substantially lower. After cooling, the reaction vessel contained a solid $\mathrm{LiBr} / \mathrm{KBr}$ block mixed with $\mathrm{C}_{x} \mathrm{~N}_{y} \mathrm{H}_{z}$ reaction products, along with black shiny flakes coating the upper internal walls. Breaking up the solid block and removing the $\mathrm{LiBr} / \mathrm{KBr}$ salt by washing in distilled water yielded a dark brown/black powder that contained a large quantity of small shiny flakes. This mixture was filtered and washed to remove residual salts. Larger flakes up to $1-5 \mathrm{~mm}$ in dimension ( $70 \mathrm{mg}$ aliquots from each run $)$ were also hand separated from the reaction products. One unexpected result was that $\mathrm{X}$-ray analysis of the powdered run products from the $\mathrm{LiBr} / \mathrm{KBr}$ salt block did not reveal the presence of any crystalline PTI $\cdot \mathrm{LiBr}$, that has previously been reported to form under similar reaction conditions. ${ }^{12-14,25}$ However, we did observe formation of this phase within the molten salt medium in experiments with the ampoule mounted vertically in our Deltech furnace. Previously, Wirnhier et al. have noted differences in the nature and morphology of PTI materials produced as a function of the reaction chamber geometry. ${ }^{14}$ 

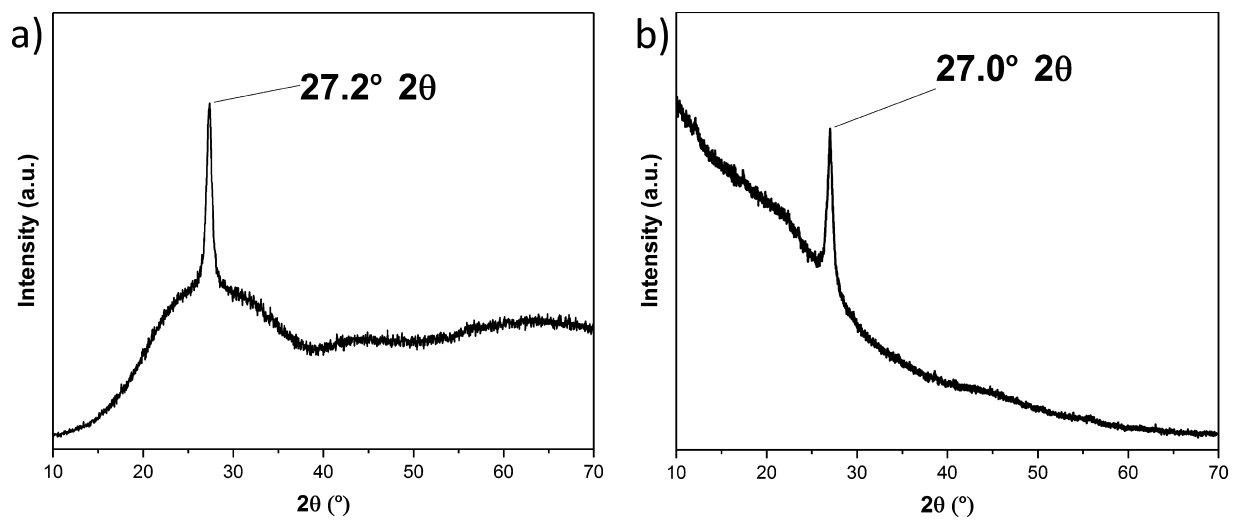

Figure 2. XRD patterns obtained for single flakes of layered carbon nitride $\left(\mathrm{gC}_{x} \mathrm{~N}_{y}\right)$ materials $(\mathrm{Cu} \mathrm{K} \alpha: l=1.5418 \AA$ ). (a) XRD pattern taken in transmission geometry while rocking the sample by $\pm 5^{\circ}$ about the incident X-ray beam axis. (b) XRD pattern taken in reflection mode with the scattering vector aligned normal to the sample surface.

\subsection{Bulk Chemical Analyses and X-ray Photoelectron} Spectroscopy. Samples of separated flakes and powdered materials from three different synthesis runs were sent to the University of Sheffield, Department of Chemistry CCIAS service for $\mathrm{CNH}$ analysis. 5-10 $\mathrm{mg}$ aliquots of each sample were catalytically combusted in a Vario Micro cube CHN/S analyser to produce $\mathrm{N}_{2}, \mathrm{CO}_{2}$ and $\mathrm{H}_{2} \mathrm{O}$ that were quantified using thermal conductivity detection. XPS measurements were carried out at UCL with a Thermo Scientific spectrometer using monochromated $\mathrm{Al} \mathrm{K} \alpha$, radiation. Samples were mounted on conductive carbon tape and a charge compensating flood-gun was applied to avoid charging effects. XPS experiments were carried out both on as-prepared samples and following $\mathrm{Ar}^{+}$ion-milling erosion to remove surface contaminant species.

2.3. XRD and HRTEM Studies. XRD patterns for sample flakes on a glass support were first obtained with $\mathrm{Cu} \mathrm{K} \alpha$ radiation $(\lambda=1.5418 \AA)$ using a Philips X-Pert diffractometer in reflection geometry, with the scattering vector aligned normal to the sample surfaces. Additional studies were carried out using individual flakes mounted with their largest dimension normal to the X-ray beam in a Stoe Stadip diffractometer, with data collected while rotating the sample by $\pm 5^{\circ}$ about the incident beam axis to introduce orientation averaging. HRTEM studies were carried out for samples prepared by sonication in methanol followed by dropping the resultant suspension onto 300 mesh gold TEM grids with a holey carbon film. TEM images, selected area diffraction (SAED) patterns and energy dispersive X-ray (EDX) imaging results were obtained using a JEOL JEM-2100 instrument.

2.4. IR, Raman and Electron Paramagnetic Resonance Spectroscopy. FTIR data were obtained by transmission through individual flakes placed on a $\mathrm{CaF}_{2}$ slide using a Bruker FTS-66v microbeam instrument. Samples of different thicknesses were examined. Unpolarized microbeam Raman spectra were recorded for individual flakes on $\mathrm{CaF}_{2}$ supports using a Renishaw InVia system with $325 \mathrm{~nm}(\mathrm{He}-\mathrm{Cd}), 514.5 \mathrm{~nm} \mathrm{Ar}^{+}$or $785 \mathrm{~nm}$ diode laser excitation. All spectra presented here were obtained with the incident beam normal to the sample flakes. Additional data obtained at different angles to the incident beam showed no differences in the relative band intensities, and the spectra did not change during rotation about the incident beam axis. Electron paramagnetic resonance (EPR) measurements were performed using a Bruker EMXplus spectrometer at 9.85 $\mathrm{GHz}$ (X-band) equipped with a 4122SHQE resonator. Spectra were acquired with a magnetic field sweep of $8 \mathrm{mT}$, microwave power $0.5 \mu \mathrm{W}$, modulation amplitude $0.1 \mathrm{mT}$ and frequency modulation $100 \mathrm{kHz}$. To minimize instabilities and drift in the applied magnetic field, the electromagnet was left to stabilize for $8 \mathrm{~h}$ prior to collection. The applied field was calibrated against the Bruker strong pitch standard $(g=2.0028)$. A single flake of the carbon nitride material was mounted with its plane parallel to the long axis of an EPR tube, which was rotated in the resonator by means of a laboratory-built goniometer (resolution $\left.\pm 5^{\circ}\right)$. Spectra were collected every $20^{\circ}$, and at every $10^{\circ}$ near the extrema. Simulations of the continuous wave-EPR spectra were carried out to determine orientation-dependent $g$-values and linewidths using the Easyspin toolbox running in Matlab. Magnetic susceptibility data shown in the Supporting Information (Figure S7) were acquired for single flakes using a Quantum Design MPMS-7 magnetometer with the field applied perpendicular to the flake surface. Temperature scans were taken upon warming from 5 to $300 \mathrm{~K}$ in 1000 and $2000 \mathrm{Oe}$ magnetic fields.

2.5. Computational Studies. DFT calculations were carried out for different model structures based on $\mathrm{gC}_{3} \mathrm{~N}_{4}$ and $\mathrm{gC}_{4} \mathrm{~N}_{3}$ motifs, implemented using the hybrid exchangecorrelation B3LYP functional ${ }^{26,29}$ within the Crystal14 code. ${ }^{28,29}$ We used $6-21 \mathrm{G}^{* *}$ Gaussian basis sets and all atoms were treated at all-electron level. We applied Monkhorst-Pack sampling of 6 and 16 for semiconducting and metallic systems, respectively, with 20 and $130 k$-points in the irreducible Brillouin zone. Truncation thresholds were set to (6 666612$)$ for the Coulomb and exchange series. SCF convergence was set to $10^{-7}$ hartree for structure optimization and to $10^{-8}$ hartree for calculation of IR and Raman spectra. The fractional coordinates and cell parameters were fully optimized within $P 1$ space group. Structure optimization was deemed to have converged when the largest gradient component was $1.2 \times 10^{-3}$ a.u. and the largest estimated nuclear displacement was $1.8 \times 10^{-3}$ a.u. Vibrational spectra were calculated within the harmonic approximation. ${ }^{30,31}$ IR intensities were evaluated using the localized Wannier function approach and Raman intensities by a coupled perturbed Hartree-Fock/Kohn-Sham method ${ }^{32}$ DFT cluster calculations implemented within Gaussian $09^{33}$ using B3LYP/ EPR-II were used to predict localized electron spin densities and hyperfine coupling (hfc's) to interpret the EPR data. ${ }^{26,27}$

\section{RESULTS AND DISCUSSION}

3.1. XRD and HRTEM. X-ray reflection data for flakes of our $\mathrm{gC}_{x} \mathrm{~N}_{y} \mathrm{~N}_{z}$ sample mounted on a glass support showed a single 

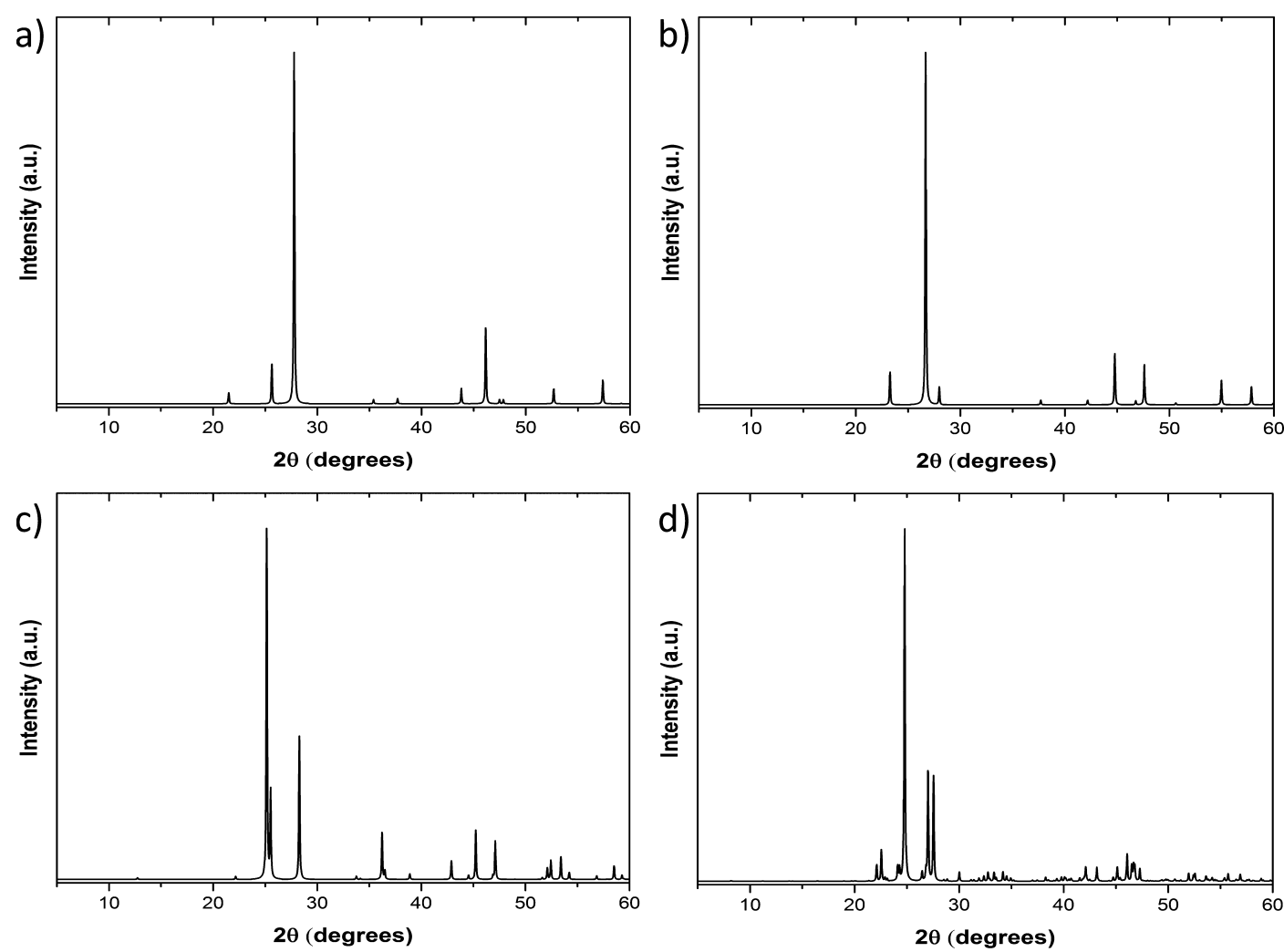

Figure 3. Calculated $\mathrm{XRD}$ patterns $\left(\mathrm{Cu} \mathrm{K} \alpha\right.$ radiation) for planar vs buckled layer configurations of triazine based $\mathrm{gC}_{3} \mathrm{~N}_{4}$ structures optimized from DFT calculations. (a) Planar layers with AB stacking. (b) Planar ABC stacking. (c) Buckled layers with AB stacking. (d) Buckled sheets with ABC stacking.

broad peak near $27^{\circ} 2 \theta(\mathrm{Cu} \mathrm{K} \alpha$ radiation) indicated a layered material with interlayer spacing $\sim 3.24 \AA$, comparable to the $d_{002}$ reflection of crystalline graphite $(d=3.28 \AA)$. Similar results were obtained in transmission experiments for a single flake obtained while rocking the sample by $\pm 5^{\circ}$ about the beam axis (Figure 2). A similar pattern was observed by Ladva et al. ${ }^{34}$ for their carbon nitride films. However, Algara-Siller et al. obtained different results for their TGCN samples prepared using a similar synthesis procedure. The background-subtracted XRD patterns reported by these authors showed a main peak at $27.2^{\circ}$ $2 \theta$ accompanied by a smaller peak near $24.4^{\circ} 2 \theta$ that they assigned to the 101 reflection of the graphitic structure, along with additional weak broad features at $\sim 50^{\circ}$ and $56^{\circ} 2 \theta .^{8}$ They refined their X-ray data within space group $P \overline{6} \mathrm{~m} 2$ consistent with $\mathrm{AB}$ stacking of the triazine-based $\mathrm{gC}_{3} \mathrm{~N}_{4}$ layers, although HRTEM image analysis of thin specimens of the same samples were interpreted to indicate $\mathrm{ABC}$ layer stacking (i.e., space group $\left.P 6_{3} \mathrm{~cm}\right)$. $^{8}$

In their XRD analyses, Algara-Siller et al. assumed a planar model for the $\mathrm{gC}_{3} \mathrm{~N}_{4}$ layers, although both their and our DFT calculations indicated that the equilibrium geometry should be buckled. ${ }^{8}$ Here we calculated XRD patterns for both $\mathrm{AB}$ and ABC stacked models of the TGCN structure, using planar and fully-relaxed buckled layer geometries (Figure 3). We note that the character of the diffraction patterns changes significantly once layer buckling is taken into account. In particular, the relative intensities of the two main peaks in the $25^{\circ}-30^{\circ} 2 \theta$ range are reversed for the buckled layer solutions that correspond to the most stable TGCN arrangements.

We also obtained powder XRD patterns for the brown/black powdered residue recovered after removing the soluble components from the solidified salt block by washing. We note that reacting DCDA in molten $\mathrm{LiBr} / \mathrm{KBr}$ is typically expected to result in formation of crystalline PTI. $\mathrm{LiBr}{ }^{12-14,25}$ That phase was found among the run products from reactions carried out in the vertical furnace geometry, that led to significantly lower yield of the shiny dark colored carbon nitride flakes. However, we found no evidence for the PTI-LiBr phase among the run products from synthesis experiments in the horizontal tube furnace. Instead, the XRD pattern showed a main broad peak at $27.2^{\circ} 2 \theta$ as observed for the larger individual flake samples, along with a shoulder at $28.2^{\circ} 2 \theta$ and weaker sharp reflections at $21^{\circ}, 24.2^{\circ}, 32.3^{\circ}$ and $27.2^{\circ} 2 \theta$ (Figure 4 ). We note that similar peaks were observed by Algara-Siller et al. for their ground "TGCN" sample in a synchrotron powder XRD experiment $(\lambda=0.827127 \AA) .{ }^{8}$ Those peaks do not match the predicted reflections for the TGCN structure. We suggest that they could correspond to an as yet unidentified carbon nitride phase that is formed during the condensation/polymerization reaction from DCDA in the molten salt medium. We note here that several new crystalline phases are now predicted theoretically to occur within the $\mathrm{C}-\mathrm{N}-\mathrm{H}$ system, containing both layered and open-framework structures based on $\mathrm{sp}^{2}$ as well as $\mathrm{sp}^{3}$ bonding. 9

As shown in previous studies, optical microscopy and SEM images reveal uniformly flat surfaces and terraced edges for the layered $\mathrm{gC}_{x} \mathrm{~N}_{y}$ structures (Figure S1). ${ }^{8,34}$ HRTEM images for uncleaved thin edge regions of our samples (Figure 5a-d) show crystalline in-plane lattice fringes, and Fourier transformation of the imaged intensity data demonstrate the characteristic $2.6 \AA$ hexagonal lattice spacing typical of graphitic carbon-based nanomaterials. ${ }^{35,36}$ SAED patterns exhibit multiple spots and the 


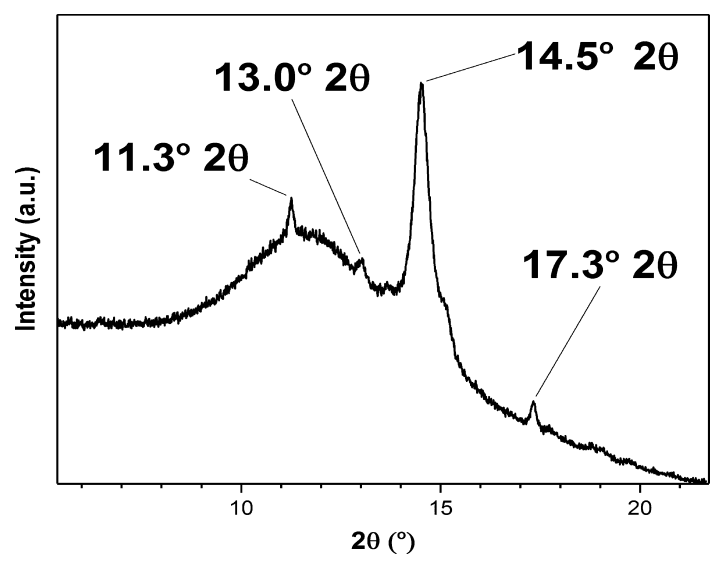

Figure 4. Powder XRD pattern of the brown/black powdered residue obtained from the solid $\mathrm{LiBr} / \mathrm{KBr}$ block after the synthesis reaction following washing to remove alkali halide components. The pattern was obtained using $\mathrm{Cu} \mathrm{K} \alpha$ radiation but $2 \theta$ values have been recalculated for $\lambda=0.827127 \AA$ for ease of comparison with previously published data recorded using a synchrotron X-ray source (ref 8 ).

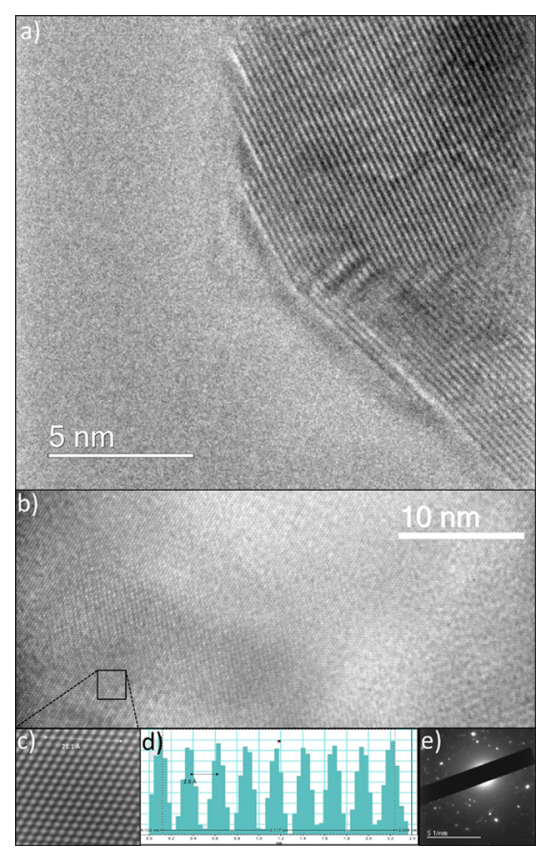

Figure 5. TEM results for uncleaved flakes of a typical layered carbon nitride sample. (a) Thin regions at the sample edges reveal lattice fringes and layering representative of crystalline material with a graphitic structure. (b) Images obtained within the flakes show evidence for overlapping domains with different crystallographic orientations. (c) Expanded filtered TEM image of one nanocrystalline region demonstrating hexagonal symmetry within the layers with a characteristic $2.6 \AA$ separation between bright spots representing 3coordinated $\mathrm{N}$ atoms. (d) A line scan across the image intensity variations used for FT determination was used to determine the intralayer atomic separation. (e) Spots appearing in SAED patterns also indicate different crystalline layer orientations sampled by the electron beam passing through the flake.

TEM imaging data exhibit moiré patterns, demonstrating the existence of overlapping graphitic layers with different crystallographic orientations (Figure $5 \mathrm{~b}, \mathrm{e}$ ).

3.2. Chemical Composition and XPS Analysis. Samples of separated flakes and powdered material obtained after washing the salt block in water were sent for $\mathrm{CNH}$ analysis
(Table 1 ). We note that only $\sim 80 \%$ of the total mass was accounted for in these analyses. Our first response was to

Table 1. The $\mathrm{C}_{x} \mathrm{~N}_{y} \mathrm{H}_{z}$ Compositions of Carbon Nitride Materials Obtained in Different Synthesis Runs, or Collected from Different Parts of the Reaction Vessel, Determined by Bulk CHN Analysis ${ }^{a}$

$\begin{array}{lccccc} & \mathrm{C}(\mathrm{wt} \%) & \mathrm{N}(\mathrm{wt} \%) & \mathrm{H}(\mathrm{wt} \%) & \text { total } & \begin{array}{c}\mathrm{C} / \mathrm{N} \\ \text { ratio }\end{array} \\ \begin{array}{c}\text { synthesis run 1 } \\ \text { (flakes) }\end{array} & 40.01 & 37.63 & 2.26 & 79.9 & 1.24: 1 \\ \begin{array}{c}\text { synthesis run 1 } \\ \text { (powder) }\end{array} & 39.03 & 37.09 & 2.40 & 78.5 & 1.23: 1 \\ \begin{array}{c}\text { synthesis run 2 } \\ \text { (flake) }\end{array} & 40.01 & 38.04 & 2.53 & 80.6 & 1.23: 1 \\ & 42.54 & 40.68 & 2.38 & 85.6 & 1.22: 1 \\ & 42.29 & 40.75 & 2.39 & 85.4 & 1.21: 1\end{array}$

${ }^{a_{T}}$ The undetermined $\sim 20 \mathrm{wt} \%$ could include $\mathrm{Br}(\sim 7 \mathrm{wt} \%)$ and/or O ( $\sim$ wt $\%$ ) components, observed by XPS analysis.

potentially assign the missing mass to $\mathrm{LiBr} / \mathrm{KBr}$ salts that were either incompletely removed by washing, or that were redeposited on the sample surface afterwards. Our EDX analyses carried out during TEM studies did reveal some $\mathrm{Br}$ signals scattered randomly thoughout the samples (Figure S2). The XPS survey spectra indicated that $\sim 1$ at. $\% \mathrm{Br}$ was present within the material (Figure 6). We did not detect the presence of $\mathrm{K}$, although its $2 \mathrm{p}_{1 / 2-3 / 2}$ doublet near $300 \mathrm{eV}$ is notoriously weak, or $\mathrm{Li} 1 \mathrm{~s}$ near $55 \mathrm{eV}$, so that it is not impossible that a very small amount of $\mathrm{Br}$ might be incorporated within or attached to the surface of the $\mathrm{gC}_{x} \mathrm{~N}_{y} \mathrm{H}_{z}$ layered sample. Our XPS results also showed that $\mathrm{O}$ atoms incorporated at the surface within the bulk might also account for some of the missing mass component. Prior to $\mathrm{Ar}^{+}$etching $\sim 15 \% \mathrm{O}$ was observed at the sample surface. Although the $\mathrm{O}$ signal decreased in intensity during ion milling, it was not completely eliminated, with $\sim 6$ at. \% recorded after this process (Figure 6). That result could indicate a small percentage of $\mathrm{O}$ atoms bound within the structure. Algara-Siller et al. reported similar results from their XPS characterization of TGCN, although their chemical analysis obtained using a CHNOS analyzer reported no evidence for oxygen component within the bulk material, and they observed $\mathrm{C}, \mathrm{N}, \mathrm{H}$ values that summed to 98.7 wt \%.

Bulk chemical analyses of our samples indicated that 2.3-2.5 wt $\% \mathrm{H}$ component was present (Table 1 ). That value is similar to that reported by Ladva et al. for thin film materials prepared using an analogous synthesis approach. Our FTIR spectroscopic results presented below clearly showed that $\mathrm{N}-\mathrm{H}$ groups were incorporated within the structure. The $\mathrm{C} / \mathrm{N}$ ratio determined by bulk analysis ranged between 1.21 and 1.24:1 for both thin film and powdered materials prepared in different synthesis experiments (Table 1). This composition is significantly more C-rich than the TGCN material described by Algara-Siller et al., although it agrees with that reported by Ladva et al. for $\mathrm{gC}_{x} \mathrm{~N}_{y} \mathrm{H}_{z}$ films deposited from vapors produced by DCDA reacted in molten $\mathrm{LiBr} / \mathrm{KBr}^{34}$ These results indicate that the structure must contain $\mathrm{C}-\mathrm{C}$ as well as $\mathrm{C}-\mathrm{N}$ bonded species within its $\mathrm{sp}^{2}$ bonded graphitic layers.

Detailed analysis of the $\mathrm{C} 1 \mathrm{~s}$ and $\mathrm{N}$ 1s XPS signals provides additional information on the local bonding environments and chemical composition of $\mathrm{C}_{x} \mathrm{~N}_{y}$ materials (Figure 6). The $\mathrm{C} 1 \mathrm{~s}$ spectra of crystalline graphite and graphene exhibit an asymmetric peak at $284.5-284.6 \mathrm{eV}$. Most samples examined 

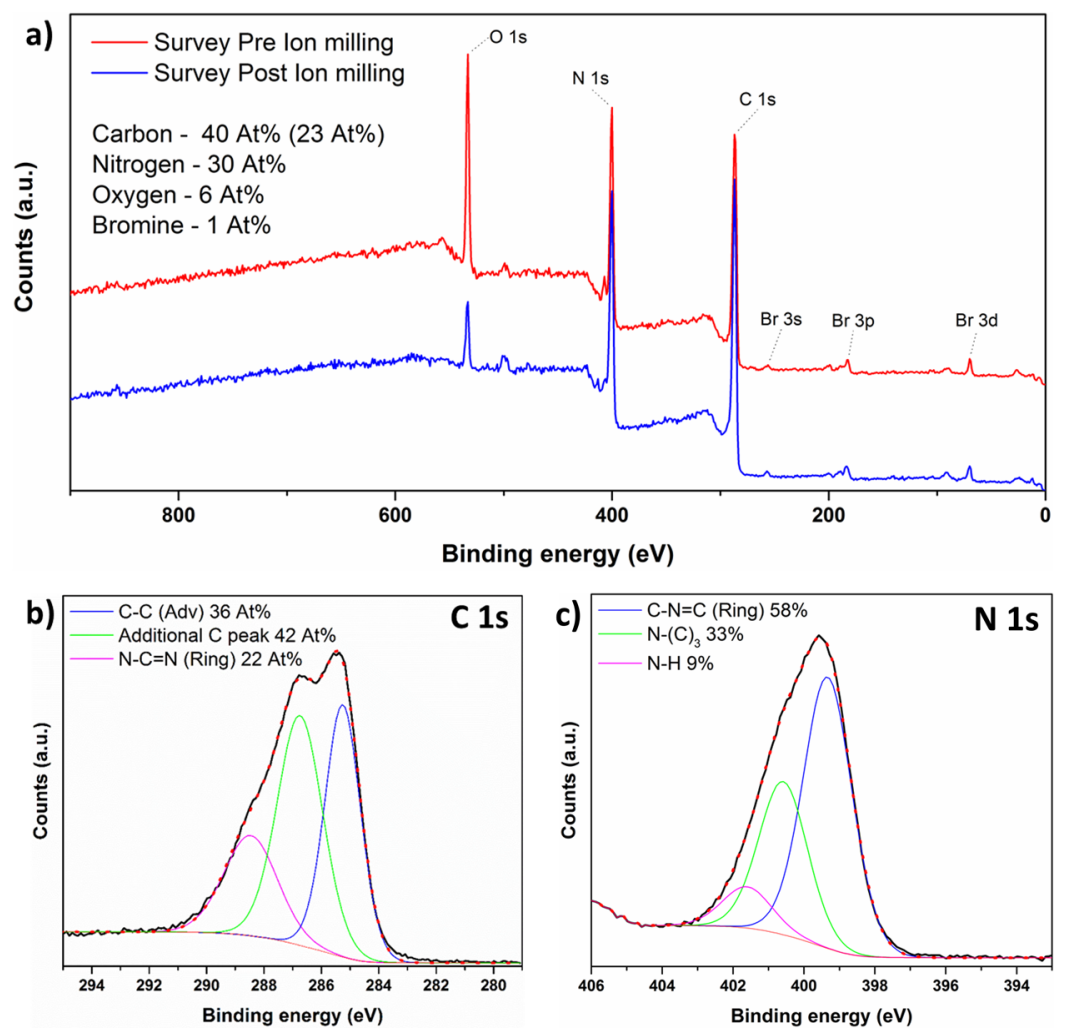

Figure 6. (a) XPS survey spectra of carbon nitride flake samples before (red) and after (blue) $\mathrm{Ar}^{+}$ion beam milling to remove surface contaminant species. The first carbon content indicated is for those $\mathrm{C}$ atoms that do not occur in "adventitious" component environments, whereas the value in brackets indicates the amount that could be assigned to adventitious carbon, but might also partially correspond to signal from the sample (see text for discussion). (b) C 1s and (c) N 1s spectra showing deconvolution into Gaussian-Lorentzian components to show individual contributions to the overall lineshape.
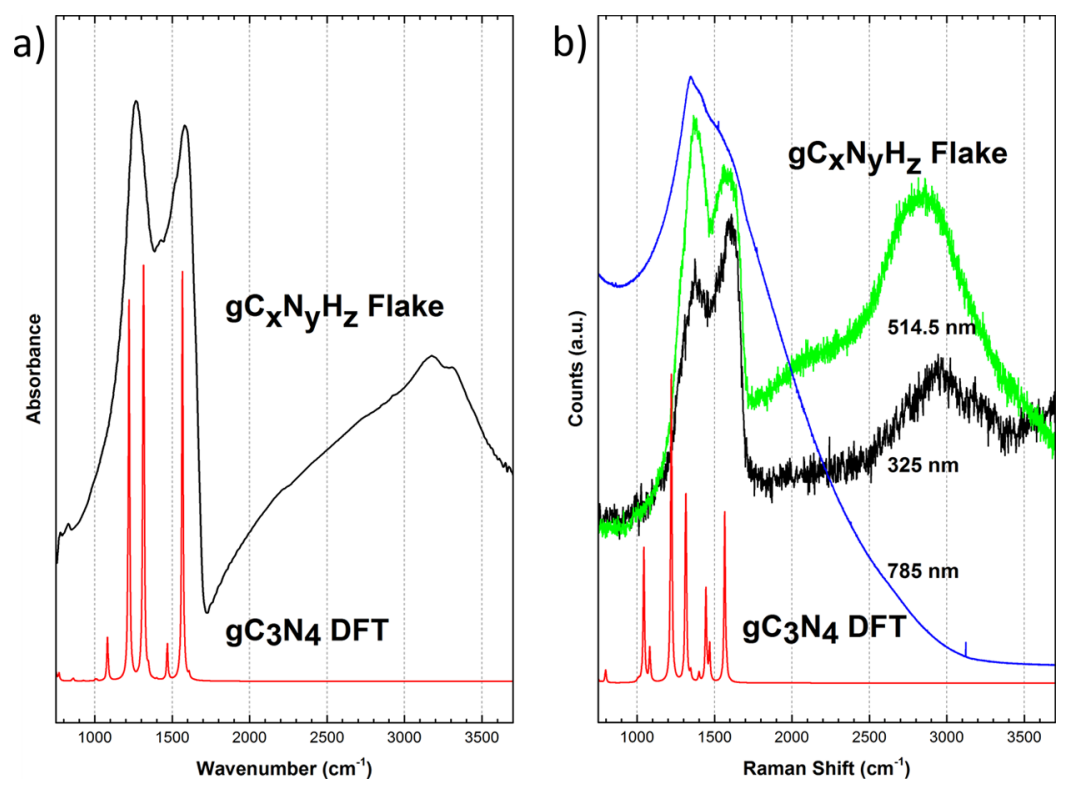

Figure 7. Experimental and calculated IR and Raman spectra for layered carbon nitride materials. (a) IR absorption spectrum obtained by transmission through a single flake of graphitic carbon nitride $\left(\mathrm{gC}_{x} \mathrm{~N}_{y} \mathrm{H}_{z}\right)$ material prepared in this study. This is compared with the result of a DFT calculation for a triazine based $\mathrm{gC}_{3} \mathrm{~N}_{4}$ layered structure. (b) Raman spectra of a typical $\mathrm{gC}_{x} \mathrm{~N}_{y} \mathrm{H}_{z}$ flake obtained with different laser excitation wavelengths (325 nm: black; $514.5 \mathrm{~nm}$ : green; $785 \mathrm{~nm}$ : blue) compared with DFT calculation for triazine based $\mathrm{gC}_{3} \mathrm{~N}_{4}$.

by XPS show a feature near $284.5-285.0 \mathrm{eV}$ that is typically assigned to "adventitious" carbon species at the sample surface, that can include oxygenated components. ${ }^{37,38}$ Graphitic and graphene oxide (GO) samples typically show a strong peak emerging at $\sim 286.1 \mathrm{eV}$ due to $\mathrm{COH}$ and $\mathrm{COC}$ species incorporated within the layered materials. ${ }^{39,40} \mathrm{~N}$-doped graphene exhibits a main peak at $284.6 \mathrm{eV}$ due to $\mathrm{sp}^{2}$-bonded atoms occurring within the majority $\mathrm{C}-(\mathrm{C})_{3}$ environments, 
while additional peaks emerging near 285.8 (shoulder) and $288.2 \mathrm{eV}$ are coincident with the appearance of a strong $\mathrm{N}$ 1s signal at $400.6 \mathrm{eV}$ and a weaker feature at $398.6 \mathrm{eV}$. ${ }^{19} \mathrm{~A} \mathrm{C} 1 \mathrm{~s}$ peak at $285.5 \mathrm{eV}$ was observed to grow as a function of $\mathrm{N}$ content in $\mathrm{N}$-doped graphene and was assigned to the emergence of $\mathrm{C}-$ $\mathrm{N}$ bonding. ${ }^{41}$ However, detailed assignment and interpretation of $\mathrm{C}$ 1s and $\mathrm{N}$ 1s XPS signals for carbon nitride compounds remains an active research problem, while many different authors have either accepted without question, or have modified and added to the suggested assignments, without fully supporting their arguments.

The $\mathrm{C}$ 1s data for our $\mathrm{gC}_{x} \mathrm{~N}_{y} \mathrm{H}_{x}$ compound exhibited two clear maxima, along with a shoulder at higher binding energy. These features were best fit using three Gaussian-Lorentzian contributions. The $288.5 \mathrm{eV}$ peak occurs at a similar position to that for $\mathrm{sp}^{2}$-bonded $\mathrm{C}$ atoms in melamine, ${ }^{42}$ and it is readily assigned to $-\mathrm{N}-\mathrm{C}=\mathrm{N}-$ bonded species that occur within the polymerized triazine rings of the gCN samples. ${ }^{43}$ A fully polymerized TGCN structure should give rise to a single $\mathrm{C}$ 1s peak near this position, whereas our data indicate three component signals (Figure 6). It would be usual to assign the $285.3 \mathrm{eV}$ component to "adventitious" carbon, and then ignore that contribution in order to obtain a $\mathrm{C} / \mathrm{N}$ ratio for the $\mathrm{gC}_{x} \mathrm{~N}_{y} \mathrm{H}_{z}$ layered structure. However, that solution would not account for our bulk chemical analyses or the results of Ladva et al., showing that the $\mathrm{C} / \mathrm{N}$ ratio is greater than expected for the $\mathrm{C}_{3} \mathrm{~N}_{4}$ stoichiometry. ${ }^{20}$ Taking account of those results then indicates that at least part of the $285.3 \mathrm{eV} \mathrm{C} 1$ s peak intensity must be due to non-adventitious carbonaceous bonding environments within the layered $\mathrm{gC}_{x} \mathrm{~N}_{y} \mathrm{H}_{z}$ materials. Assignment of the $286.7 \mathrm{eV} \mathrm{C} \mathrm{1s}$ component is likewise unclear. This feature was observed in the XPS data of Algara-Siller et al., where it was suggested to arise from $-\mathrm{C} \equiv \mathrm{N}$ (nitrile) functional groups. We observed no evidence for these species by IR spectroscopy (Figure 7a). However, N-doped graphene exhibits a secondary $\mathrm{C} 1 \mathrm{~s}$ peak at the same position. ${ }^{44}$ We propose that the feature observed in our study could be associated with $\mathrm{sp}^{2} \mathrm{C}$ atoms bound to either one or two $\mathrm{N}$ atoms within the graphene-like layers of our $\mathrm{gC}_{x} \mathrm{~N}_{y} \mathrm{H}_{z}$ materials.

The $\mathrm{N}$ 1s profile is best fit using three Gaussian-Lorentzian components (Figure 6c). The main contribution in the N1s profile at $399.3 \mathrm{eV}$ is readily assigned to $\mathrm{sp}^{2}$-bonded $-\mathrm{C}-\mathrm{N}=$ $\mathrm{C}-$ environments such as those expected for triazine-based or related $\mathrm{C}_{x} \mathrm{~N}_{y}$ ring units. A similar peak has been documented for both melamine ${ }^{42}$ and $\mathrm{N}$-doped graphene. ${ }^{44}$ The $400.6 \mathrm{eV}$ contribution is assigned to $\mathrm{N}-(\mathrm{C})_{3}$ bridging environments between the rings, as proposed by Algara-Siller et al., although the intensity ratio between the two features does not correspond to that expected for the TGCN structure. The minor peak at $401.6 \mathrm{eV}$ is typically associated with $\mathrm{N}-\mathrm{H}$ bonded environments, that have been confirmed to be present in our study by FTIR spectroscopy (Figure 7a).

3.3. Vibrational Spectroscopy and DFT Modelling. Microbeam IR transmission spectra obtained through individual flakes of our $\mathrm{gC}_{x} \mathrm{~N}_{y}$ materials are dominated by strong in-plane $\mathrm{C}-\mathrm{N} / \mathrm{C}-\mathrm{C}$ stretching modes at 1276 and $1590 \mathrm{~cm}^{-1}$ (Figure 7a). A small absorption maximum can be observed between the two main bands at $1420 \mathrm{~cm}^{-1}$, and a weak sharp peak occurs at $\sim 700 \mathrm{~cm}^{-1}$. We note an unusual "dip" in the IR spectra just above the intralayer vibrational modes that resembles the Fano resonance lineshape observed for graphene and related $2 \mathrm{D}$ materials, caused by coupling between the IR-active vibrational modes and a broad spectrum of electronic excitations. ${ }^{45}$ Our IR spectra also exhibit absorption maxima at 3179 and $3311 \mathrm{~cm}^{-1}$ that resemble the $\mathrm{N}-\mathrm{H}$ stretching modes observed for crystalline $\mathrm{PTI} \cdot \mathrm{LiBr}$ and $\mathrm{PTI} \cdot \mathrm{LiCl}$ compounds (Figure S3) ${ }^{13,14}$ However, we found no evidence for the presence of PTI phases from our XRD or TEM studies, indicating that the $\mathrm{N}-\mathrm{H}$ stretching vibrations are intrinsic to the $\mathrm{gC}_{x} \mathrm{~N}_{y} \mathrm{H}_{z}$ flakes. Another possibility might be that these modes are related to $\mathrm{O}-$ $\mathrm{H}$ stretching vibrations, that could be associated with the small proportion of $\mathrm{O}$ atoms concentrated at the surface and detected by XPS measurements. However, the $\mathrm{N}-\mathrm{H}$ absorptions are different from the broad $\mathrm{O}-\mathrm{H}$ stretching feature observed for GO materials (Figure S3).

We also obtained microbeam Raman scattering spectra for our $\mathrm{gC}_{x} \mathrm{~N}_{y} \mathrm{H}_{z}$ samples using laser excitation wavelengths ranging from the near-IR $(785 \mathrm{~nm})$ to visible $(514.5 \mathrm{~nm})$ and UV $(325$ $\mathrm{nm}$ ) (Figure 7b). The appearance of the spectra depends on the excitation wavelength, as has been observed previously for Cgraphite, graphene and $\mathrm{gC}_{x} \mathrm{~N}_{y}$ samples. ${ }^{46-49}$ It is known that different vibrational modes can exhibit resonant behaviour with various parts of the electronic absorption manifold, and the resulting spectra can probe different spatial extents of vibrational coherence and structural ordering within the samples. ${ }^{10}$ In general, UV excitation tends to favour more localized vibrations whereas near-IR Raman studies probe responses over longer length scales, that could include structurally disordered regions. Our UV-Raman data exhibit main peaks near 1700 and 1300 $\mathrm{cm}^{-1}$ that resemble the "G" and "D" modes of graphite and graphene materials, ${ }^{46-49}$ and they can be assigned to $\mathrm{C}-\mathrm{N}$ and/ or $\mathrm{C}-\mathrm{C}$ stretching vibrations of the $\mathrm{sp}^{2}$-bonded network. As the excitation wavelength moves into the near-IR range the phonon response becomes broadened and loses its intrinsic structure (Figure $7 \mathrm{~b}$ ). Our data indicate wavelength dispersion occurring for the fundamental $\left(1200-1700 \mathrm{~cm}^{-1}\right)$ stretching modes as well as the Raman active overtone in the $2500-3500 \mathrm{~cm}^{-1}$ region. ${ }^{47,48,50} \mathrm{~N}$-doped graphene and graphite samples exhibit IR absorption bands in the $1200-1700 \mathrm{~cm}^{-1}$ region that resemble the $G$ and $D$ band profile observed in the Raman spectra of structurally disordered or nanocrystalline pure carbon materials. ${ }^{46,48,51,52}$ Rodil et al. ${ }^{46,53}$ have analyzed the effects giving rise to the IR band profiles obtained from different types of amorphous $\mathrm{C}_{x} \mathrm{~N}_{y}$ films.

To aid our understanding of the vibrational spectra and structural nature of the layered carbon nitride materials prepared here, we carried out DFT calculations for series of models representing structural motifs that might be present among the $\mathrm{gC}_{x} \mathrm{~N}_{y} \mathrm{H}_{z}$ layered phases (Figures $7 \mathrm{a}, \mathrm{b} ; 8 \mathrm{a}, \mathrm{b}$ ). We began with a single sheet of ideal triazine based $\mathrm{gC}_{3} \mathrm{~N}_{4}$ (TGCN), that was fully relaxed into its buckled conformation. The simulated IR spectrum for this material shown in Figure 7a displays three main peaks in the $\mathrm{C}-\mathrm{N}$ stretching region between 1200 and $1600 \mathrm{~cm}^{-1}$. The predicted strong in-plane $\mathrm{C}-\mathrm{N}$ modes agree generally with the dominant IR bands observed for our sample, although the $\mathrm{N}-\mathrm{H}$ stretching features are absent. We have compared our simulated spectrum with IR data for nanocrystalline $\mathrm{H}$-free $\mathrm{gC}_{3} \mathrm{~N}_{4}$ reported by Kouvetakis et al. ${ }^{2}$ That compound exhibits a single broad band between 1100 and $1700 \mathrm{~cm}^{-1}$, with its main peak at approximately $1300 \mathrm{~cm}^{-1}$, covering the range of our DFT results. Broadening in the experimental spectrum might occur due to finite particle and domain size effects, along with local differences in layer buckling and stacking patterns within the nanoparticles. However, the $\mathrm{gC}_{3} \mathrm{~N}_{4}$ layered model predicts the occurrence of strong Raman active modes at $1040-1050 \mathrm{~cm}^{-1}$ associated with symmetric 

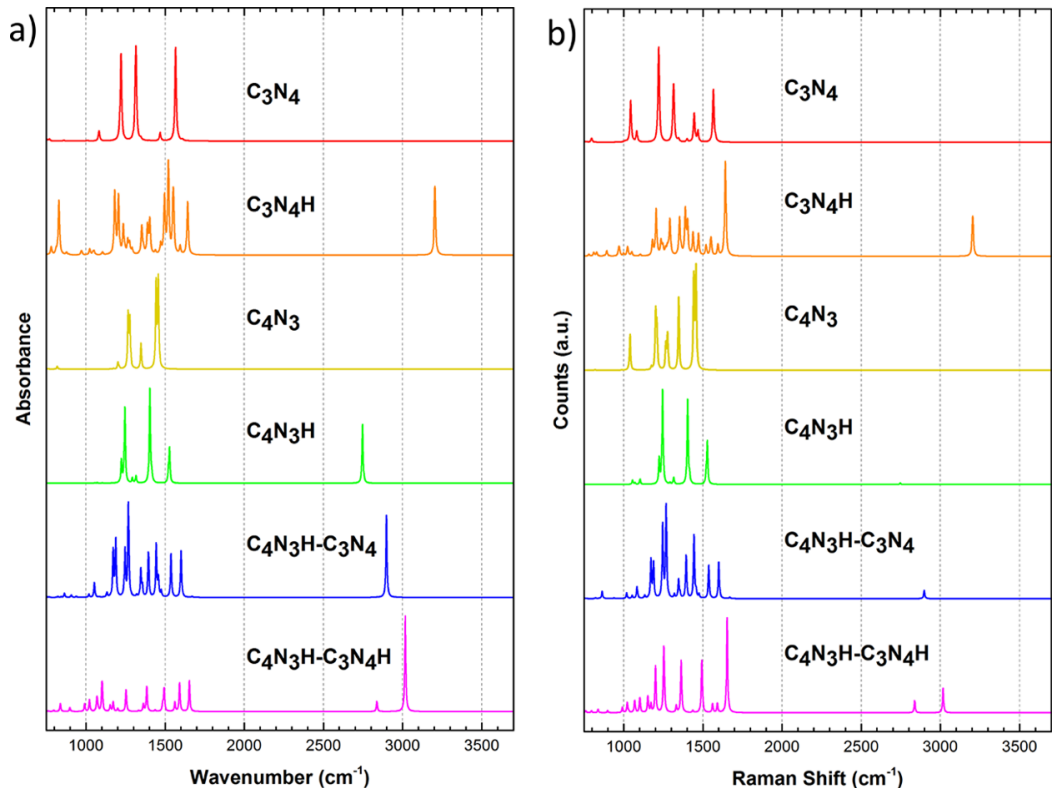

Figure 8. Simulated IR and Raman spectra for different DFT model structures based on $\mathrm{C}_{3} \mathrm{~N}_{4}, \mathrm{C}_{4} \mathrm{~N}_{3}, \mathrm{C}_{3} \mathrm{~N}_{4} \mathrm{H}$ and $\mathrm{C}_{4} \mathrm{~N}_{3} \mathrm{H}$ units. (a) Calculated IR spectra for different model sheet structures. (b) Calculated Raman spectra for the corresponding model structures.

breathing vibrations of the $\mathrm{C}_{3} \mathrm{~N}_{3}$ triazine rings, that are absent from our data (Figure $7 \mathrm{~b}$ ). That observation caused us to examine the nature of the vibrational mode symmetries in more detail, and to construct further models that could account for the observed spectra.

Analysis of the IR-active normal modes of our simulated $\mathrm{gC}_{3} \mathrm{~N}_{4}$ (TGCN) shows that the $1566 \mathrm{~cm}^{-1}$ peak arises from inphase stretching of two parallel $\mathrm{C}-\mathrm{N}$ bonds within the same triazine ring, combined with parallel out-of-phase movement of the remaining $\mathrm{N}$ atom that connects them together. For convenience, we refer to these two-coordinated $\mathrm{N}$ atoms within the triazine ring as $\mathrm{N}^{(2)}$, and denote the bridging threecoordinated $\mathrm{N}$ atoms that link the triazine units as $\mathrm{N}^{(3)}$. The $\sim 1314 \mathrm{~cm}^{-1}$ vibrations involve the same sets of bonds and atoms, but the $\mathrm{C}-\mathrm{N}^{(2)}$ stretching is out-of-phase. The $\sim 1220$ $\mathrm{cm}^{-1}$ peak is a doublet, constituting in- and out-of-phase stretching combinations of the $\mathrm{C}-\mathrm{N}^{(3)}$ bonds.

We further developed our modelling analysis by assessing how to introduce $\mathrm{N}-\mathrm{H}$ into our model structure, while removing the high local symmetry giving rise to the $1044 \mathrm{~cm}^{-1}$ Raman active mode (Figure 8a,b). A suitable model could be found for the $\mathrm{gC}_{3} \mathrm{~N}_{4}$ framework by introducing a neutral $\mathrm{H}$ atom that forms a bond with a $\mathrm{N}^{(2)}$ atom in the triazine ring (Figure 9). We note that this structural model does not imply any "protonation" of the $\mathrm{gC}_{3} \mathrm{~N}_{4}$ layers. Instead, it corresponds to generation of a chemically reduced $\mathrm{gC}_{3} \mathrm{~N}_{4} \cdot \mathrm{H}$ structure, with the reducing $\mathrm{H}$ atom bonded to the triazine ring and donating an unpaired electron to the system. Such radical species are well documented to occur among various $\mathrm{N}$-containing compounds, ${ }^{3}$ including carbon nitrides. ${ }^{4}$ Within such a model, the unpaired electron could occur as a polaronic state displaying localized paramagnetism, or might contribute to a conduction band. Our DFT calculations predict that a dispersionless polaronic state constitutes the most stable configuration, $\sim 1 \mathrm{eV}$ below the $\mathrm{CB}$ minimum, with the spin density localized in the ring to which the $\mathrm{H}$ is bound. We found that these polaronic defects were stable as the $\mathrm{H}$-content was varied, from a single $\mathrm{H}$-atom per 12 triazine rings, to a maximum in which all the triazine rings bear $1 \mathrm{H}^{\bullet}$ atom. A reduced $\mathrm{gC}_{3} \mathrm{~N}_{4}$ material with such a $\mathrm{gC}_{3} \mathrm{~N}_{4} \cdot \mathrm{H}$

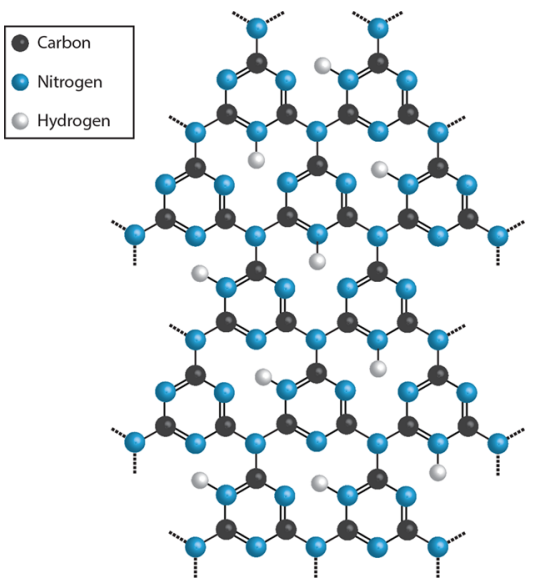

Figure 9. Schematic diagram of a neutral g- $\mathrm{C}_{3} \mathrm{~N}_{4} \cdot \mathrm{H}$ layered model, with one $\mathrm{H}$ atom attached to each triazine ring.

stoichiometry would constitute a system with a very high density of localized polarons $\left(1.78 \times 10^{-6} \mathrm{e} / \mu \mathrm{m}^{2}\right)$. A metallic conductive state was predicted to occur at only $\sim 0.26 \mathrm{eV}$ above the ground state, indicating that a temperature-dependent equilibrium between localized paramagnetic and conductive states might be established for $\mathrm{gC}_{3} \mathrm{~N}_{4} \cdot \mathrm{H}$ doped materials. We describe the results of EPR experiments and magnetic susceptibility studies to investigate the magnetic properties of our synthesized $\mathrm{gC}_{x} \mathrm{~N}_{y} \mathrm{H}_{z}$ materials below.

Although the reduced $\mathrm{gC}_{3} \mathrm{~N}_{4} \cdot \mathrm{H}$ model yields a system that could satisfy the constraints of the experimental IR and Raman observations, our analytical measurements now indicate that the prepared materials were significantly more C-rich than the $\mathrm{C}_{3} \mathrm{~N}_{4}$ stoichiometry. To introduce the effects of this observation into our DFT studies, we constructed a layered model with $\mathrm{C}_{4} \mathrm{~N}_{3}$ stoichiometry, with triazine $\left(\mathrm{C}_{3} \mathrm{~N}_{3}\right)$ rings linked by bridging $\mathrm{C}^{(3)}$ rather than $\mathrm{N}^{(3)}$ atoms (Figure 1c). Although $\mathrm{gC}_{4} \mathrm{~N}_{3}$ structures have been predicted theoretically to occur, no such material has been demonstrated experimentally. ${ }^{22-24}$ These would have unsaturated valency containing unpaired electrons, and are 

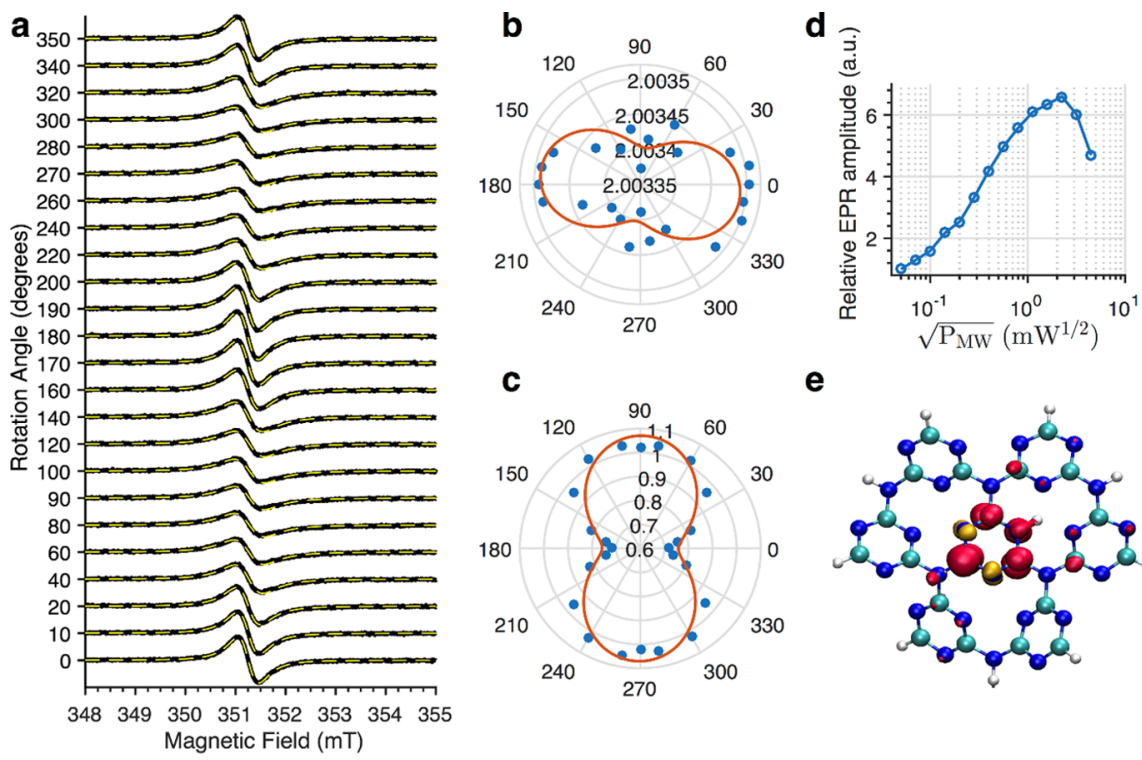

C

e
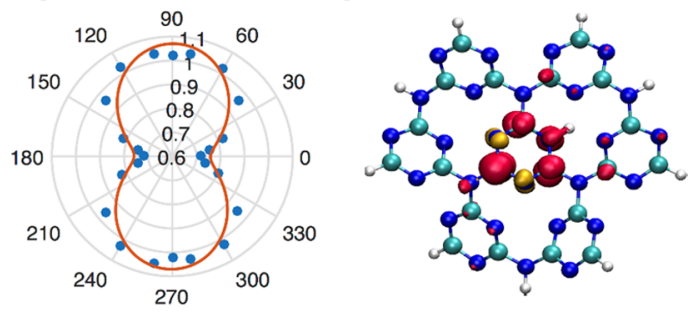

Figure 10. EPR spectrum of a single flake of a graphitic carbon nitride $\left(\mathrm{gC}_{x} \mathrm{~N}_{y} \mathrm{H}_{z}\right)$ sample prepared in this study. (a) Orientation dependence of the EPR data recorded at $9.85 \mathrm{GHz}$ (black lines) overlain with simulation results (dashed yellow lines. (b) Dependence of the $g$-values extracted from the simulations (blue circles) as a function of the relative orientation and fit (red line.) (c) Dependence of the EPR linewidth extracted from the simulations (blue circles) superimposed on the relative orientation and fit (red line). (d) Dependence of the peak-to-peak amplitude of the EPR signal as a function of microwave power $\left(P_{\mathrm{MW}}\right)$. (e) Unpaired electron spin density surfaces $\left(0.04 \mathrm{e} / \mathrm{au}^{3}\right)$ calculated for the indicated radical species superimposed on the optimized DFT structure. Red and gold surfaces indicate positive and negative spin density, respectively, and their relative sizes indicate the amount of unpaired spin density.

predicted to exhibit interesting and potentially useful "halfmetallic" properties. ${ }^{5}$ We calculated the IR and Raman spectra for $\mathrm{gC}_{4} \mathrm{~N}_{3}$ layers, as well as the "reduced" form, $\mathrm{gC}_{4} \mathrm{~N}_{3} \mathrm{H}$. The addition of neutral $\mathrm{H}$ to form $\mathrm{gC}_{4} \mathrm{~N}_{3} \mathrm{H}$ motifs generates a chargebalanced system with no unpaired electrons. Although the simulated Raman spectrum for $\mathrm{gC}_{4} \mathrm{~N}_{3}$ displays the characteristic symmetric ring-breathing mode at $\sim 1040 \mathrm{~cm}^{-1}$, forming $\mathrm{gC}_{4} \mathrm{~N}_{3} \mathrm{H}$ removes this feature as observed previously for our $\mathrm{gC}_{3} \mathrm{~N}_{4} \cdot \mathrm{H}$ model structure.

We then examined further structures containing triazine $\left(\mathrm{C}_{3} \mathrm{~N}_{3}\right)$ rings linked by both $\mathrm{N}^{(3)}$ and $\mathrm{C}^{(3)}$ atoms, that could provide models for graphene-like sheets with $\mathrm{gC}_{3} \mathrm{~N}_{4}-\mathrm{C}_{4} \mathrm{~N}_{3}$ compositions (Figure 8a,b). We also included $\mathrm{H}$ in the structures investigated. Our first model represented $\mathrm{gC}_{4} \mathrm{~N}_{3} \mathrm{H}-$ $\mathrm{C}_{3} \mathrm{~N}_{4}$, with added $\mathrm{H}$-atoms bonded to ring $\mathrm{N}^{(2)}$ atoms on $50 \%$ of the rings, and $\mathrm{N}-\mathrm{H}$ bonds aligned to point towards an $\mathrm{N}^{(3)}$ bridging atom across the layer "void". That stoichiometry corresponds to a valency-saturated system, without unpaired electrons. Analysis of the atomic displacements for the calculated vibrational modes shows that the two Raman active ring-breathing modes have very low intensity, and might not be detected experimentally. Our final model represented a $\mathrm{gC}_{4} \mathrm{~N}_{3} \mathrm{H}-\mathrm{C}_{3} \mathrm{~N}_{4} \cdot \mathrm{H}$ layer, in which all the rings have an $\mathrm{H}$ atom attached and $\mathrm{N}-\mathrm{H}$ groups occur in two different environments, pointing across the voids at either bridging $\mathrm{N}^{(3)}$ or $\mathrm{C}^{(3)}$ atoms. That structure definitely eliminates the occurrence of a $\sim 1040$ $\mathrm{cm}^{-1}$ Raman mode, while the simulated IR spectrum reveals a double peak in the $\mathrm{N}-\mathrm{H}$ stretching region at 2880 and 3020 $\mathrm{cm}^{-1}$, as is observed experimentally (Figure 7a).

3.4. EPR Spectroscopy, Paramagnetic Defects and Magnetic Properties. We carried out EPR studies on single flakes of our $\mathrm{gC}_{x} \mathrm{~N}_{y} \mathrm{H}_{z}$ material at room temperature to investigate the presence of localized paramagnetic electron species (Figure 10). Spectra were recorded from samples of $\mathrm{gC}_{x} \mathrm{~N}_{y} \mathrm{H}_{z}$ flakes over a full rotation $\left(360^{\circ}\right)$ in $20^{\circ}$ increments or $10^{\circ}$ near the turning points. The spectra at $0^{\circ}$ and $180^{\circ}$ correspond to the flake oriented parallel $\left( \pm 5^{\circ}\right)$ to the applied magnetic field, whereas spectra at $90^{\circ}$ and $270^{\circ}$ correspond to the flake held perpendicular $\left( \pm 5^{\circ}\right)$ to the field. The dependence of $g$-values as a function of the relative orientation $\left( \pm 5^{\circ}\right)$ between the sample flake and the applied magnetic field is also shown. The $g$ values showed a small dependence on orientation varying between $g=2.0035$ for parallel and 2.0034 for perpendicular orientation. However, the periodic behaviour observed throughout a full rotation indicates orientationdependent $g$-anisotropy. The EPR linewidth shows a more marked dependence on the sample orientation in the applied magnetic field with values of 0.7 and $1.0 \mathrm{mT}$ recorded for parallel versus perpendicular orientations. It is likely that the linewidth is due to unresolved hfc interactions $(A)$ between the unpaired electron and the neighbouring magnetic nuclei $(\mathrm{N}$ and $\mathrm{H})$. The red line in Figure 10b,c represents a fit according to: $A^{2}=A_{\perp}^{2} \sin ^{2}$ $\theta+A_{\|}^{2} \cos ^{2} \theta$, where $A_{\perp}$ and $A_{\|}$are the linewidths at $0^{\circ}$ and $90^{\circ}$ and $\theta$ is the angle between the applied magnetic field and a vector normal to the planar sheets, that is expected to contain the $\mathrm{p}_{z}$ orbital of a localized unpaired electron species, as found in organic radical species containing $\mathrm{N}$-atoms including neutral flavin radicals. ${ }^{54}$ We note that the EPR signal saturates readily at room temperature, that is also typical for organic radicals. In contrast to a recent study on a related carbon nitride material, ${ }^{55}$ no electron spin echo was observed. This indicates that $T_{2}^{*}$ is short in the system studied here that could indicate a high local concentration of spins.

We carried out DFT calculations for a molecular model based on a reduced triazine unit $\left(\mathrm{C}_{3} \mathrm{~N}_{3} \cdot \mathrm{H}\right)$ surrounded by $\mathrm{C}_{3} \mathrm{~N}_{3}$ groups to study the potential distribution of unpaired spin densities on atoms around the $\mathrm{C}_{3} \mathrm{~N}_{3}$ ring (Figure 10e, Table S1). The unpaired spin densities were found to be concentrated on the $3 \mathrm{C}$ atoms. However, given that $99 \%$ of these are ${ }^{12} \mathrm{C}$ nuclei, this has no appreciable impact on the observed EPR spectrum. 
However, the $3 \mathrm{~N}$ atoms $\left(>99 \%{ }^{14} \mathrm{~N}\right)$ in the reduced ring also have significant spin densities. These are predicted to have axial hfc's with the largest component perpendicular to the nearly planar $\mathrm{gC}_{3} \mathrm{~N}_{4}$ units, consistent with our observation of the largest EPR linewidths when the plane of the flake is perpendicular to the magnetic field (Figure 10, Table S1). In related organic systems such as triphyrins, tautomerism allows the proton to hop between the three neighbouring $\mathrm{N}$ atoms, giving an unusually broad NMR spectrum at room temperature. ${ }^{56}$ Analogous rapid $\mathrm{H}^{\bullet}$ transfer could also occur in the radicals proposed here, reducing the $\mathrm{N}$ hfc's by approximately a factor of three and potentially contributing to the fast $T_{2}^{*}$.

\section{CONCLUSIONS}

We prepared layered carbon nitride samples from DCDA in a molten salt $(\mathrm{LiBr} / \mathrm{KBr})$ flux inside sealed glass ampoules at $400-600{ }^{\circ} \mathrm{C}$. Dark-colored shiny flakes were either deposited on the walls of the container or formed within the molten salt. Synthesis of analogous materials under similar conditions have been reported previously to yield either crystalline triazinebased graphitic carbon nitride 8 (TGCN) $\mathrm{gC}_{3} \mathrm{~N}_{4}$, or nanocrystalline flakes with much higher $\mathrm{C} / \mathrm{N}$ ratio $(\sim 1.2: 1)$ deposited from the vapor phase. ${ }^{34}$ Our results agreed more closely with the second study. However, our experiments also indicate that the reaction products are highly dependent on details of the synthesis conditions, including furnace geometry. Our XRD and HRTEM results confirmed the graphitic structure of the flakes. The presence of moire patterns and multiple diffraction spots in our HRTEM studies indicated overlapping layers with different orientations or domain structures. Bulk chemical analyses showed a composition that was significantly more C-rich than $\mathrm{gC}_{3} \mathrm{~N}_{4}$, with $\mathrm{C} / \mathrm{N}$ ratio $\sim 1.22$ compared with 0.75 expected for $\mathrm{gC}_{3} \mathrm{~N}_{4}$. That result corresponds to observations made by Ladva et al., ${ }^{34}$ but differs from the results of Algara-Siller et al. ${ }^{8}$ for materials prepared using similar techniques. Analysis of the $\mathrm{C} 1 \mathrm{~s}$ and $\mathrm{N} 1 \mathrm{~s}$ XPS signals indicated that $\mathrm{sp}^{2}$-bonded domains with mixed $-\mathrm{C}=\mathrm{C}-$ and $-\mathrm{C}-\mathrm{N}=$ bonding is likely to present among the layered compounds. Such materials are expected to have interesting and potentially useful electronic and magnetic properties. The presence of $\mathrm{H}^{\bullet}$ defects could lead to localized unpaired electron spins or conducting domains. Taken together, the results from this and previous studies indicate that a range of $\mathrm{gC}_{x} \mathrm{~N}_{y}$ solid solutions or nanocomposite materials with tunable electronic properties might be produced by scalable thin film production techniques, by adjustment of the synthesis and deposition conditions.

\section{ASSOCIATED CONTENT}

\section{S Supporting Information}

The Supporting Information is available free of charge on the ACS Publications website at DOI: 10.1021/acs.jpcc.8b07972.

Additional data including IR, Raman spectroscopy, SEM, EDX and optical microscopy images (PDF)

\section{AUTHOR INFORMATION}

\section{Corresponding Authors}

*E-mail: theo.suter.14@ucl.ac.uk (T.S.).

*E-mail: p.f.mcmillan@ucl.ac.uk (P.F.M.).

\section{ORCID $\odot$}

Christopher A. Howard: 0000-0003-2550-0012

Paul F. McMillan: 0000-0002-5298-780X

\section{Notes}

The authors declare no competing financial interest.

\section{ACKNOWLEDGMENTS}

This work was supported by EPSRC grant EP/L017091/1 "Carbon Nitrides: Metal-free Materials for Energy Applications" and by the EU Graphene Flagship under Horizon 2020 research and innovation program grant agreement no. 696656Graphene Core 1. Calculations were performed through membership of the UK's HEC Materials Chemistry Consortium, funded by EPSRC (EP/L000202), using the ARCHER UK National Supercomputing Service (http://www.archer.ac. uk). The authors also acknowledge use of UCL's High Performance Computing Facility Grace (Grace@UCL), and associated support services. H.N. is supported by a Program for Leading Graduate Schools "Integrative Graduate Education and Research in Green Natural Sciences”, MEXT (Ministry of Education, Culture, Sports, Science and Technology), Japan.

\section{REFERENCES}

(1) Todd, M.; Kouvetakis, J.; Groy, T. L.; Chandrasekhar, D.; Smith, D. J.; Deal, P. W. Novel synthetic routes to carbon nitride. Chem. Mater. 1995, 7, 1422-1426.

(2) Kouvetakis, J.; Todd, M.; Wilkens, B.; Bandari, A.; Cave, N. Novel Synthetic Routes to Carbon-Nitrogen Thin Films. Chem. Mater. 1994, $6,811-814$

(3) Gracia, J.; Kroll, P. Corrugated layered heptazine-based carbon nitride: the lowest energy modifications of $\mathrm{C} 3 \mathrm{~N} 4$ ground state. J. Mater. Chem. 2009, 19, 3013-3019.

(4) Schwarzer, A.; Saplinova, T.; Kroke, E. Tri-s-triazines (sheptazines)-From a mystery molecule to industrially relevant carbon nitride materials. Coord. Chem. Rev. 2013, 257, 2032-2062.

(5) Teter, D. M.; Hemley, R. J. Low-compressibility carbon nitrides. Science 1996, 271, 53-55.

(6) Lowther, J. E. Relative stability of some possible phases of graphitic carbon nitride. Phys. Rev. B: Condens. Matter Mater. Phys. 1999, 59, $11683-11686$.

(7) Ortega, J.; Sankey, O. F. Relative stability of hexagonal and planar structures of hypotheticalC3N4solids. Phys. Rev. B: Condens. Matter Mater. Phys. 1995, 51, 2624-2627.

(8) Algara-Siller, G.; Severin, N.; Chong, S. Y.; Björkman, T.; Palgrave, R. G.; Laybourn, A.; Antonietti, M.; Khimyak, Y. Z.; Krasheninnikov, A. V.; Rabe, J. P.; et al. Triazine-based graphitic carbon nitride: a twodimensional semiconductor. Angew. Chem., Int. Ed. Engl. 2014, 53, $7450-7455$.

(9) Pickard, C. J.; Salamat, A.; Bojdys, M. J.; Needs, R. J.; McMillan, P. F. Carbon nitride frameworks and dense crystalline polymorphs. Phys. Rev. B 2016, 94, 094104.

(10) McMillan, P. F.; Lees, V.; Quirico, E.; Montagnac, G.; Sella, A.; Reynard, B.; Simon, P.; Bailey, E.; Deifallah, M.; Corà, F. Graphitic carbon nitride $\mathrm{C} 6 \mathrm{~N} 9 \mathrm{H} 3 \cdot \mathrm{HCl}$ : Characterisation by UV and near-IR FT Raman spectroscopy. J. Solid State Chem. 2009, 182, 2670-2677.

(11) Zhang, Z.; Leinenweber, K.; Bauer, M.; Garvie, L. A. J.; McMillan, P. F.; Wolf, G. H. High-Pressure Bulk Synthesis of Crystalline C6N9H3·HCl: A Novel C3N4Graphitic Derivative. J. Am. Chem. Soc. 2001, 123, 7788-7796.

(12) Bojdys, M. J.; Müller, J.-O.; Antonietti, M.; Thomas, A. Ionothermal synthesis of crystalline, condensed, graphitic carbon nitride. Chem.-Eur. J. 2008, 14, 8177-8182.

(13) Chong, S. Y.; Jones, J. T. A.; Khimyak, Y. Z.; Cooper, A. I.; Thomas, A.; Antonietti, M.; Bojdys, M. J. Tuning of gallery heights in a crystalline 2D carbon nitride network. J. Mater. Chem. A 2013, 1, 11021107.

(14) Wirnhier, E.; Döblinger, M.; Gunzelmann, D.; Senker, J.; Lotsch, B. V.; Schnick, W. Poly(triazine imide) with Intercalation of Lithium and Chloride Ions [(C3N3)2(NHxLi1-x)3. LiCl]: A Crystalline 2D Carbon Nitride Network. Chem.-Eur. J. 2011, 17, 3213-3221. 
(15) Kroke, E.; Schwarz, M. Novel group 14 nitrides. Coord. Chem. Rev. 2004, 248, 493-532.

(16) Cooper, A. I.; Bojdys, M. J. Carbon nitride vs. graphene - now in 2D! Mater. Today 2014, 17, 468-469.

(17) Deng, Y.; Xie, Y.; Zou, K.; Ji, X. Review on recent advances in nitrogen-doped carbons: preparations and applications in supercapacitors. J. Mater. Chem. A 2016, 4, 1144-1173.

(18) Hou, Y.; Li, J.; Wen, Z.; Cui, S.; Yuan, C.; Chen, J. N-doped graphene/porous g-C3N4 nanosheets supported layered-MoS2 hybrid as robust anode materials for lithium-ion batteries. Nano Energy 2014, $8,157-164$.

(19) Lv, R.; Li, Q.; Botello-Méndez, A. R.; Hayashi, T.; Wang, B.; Berdemir, A.; Hao, Q.; Elías, A. L.; Cruz-Silva, R.; Gutiérrez, H. R.; et al. Nitrogen-doped graphene: beyond single substitution and enhanced molecular sensing. Sci. Rep. 2012, 2, 586.

(20) Wang, Y.; Shao, Y.; Matson, D. W.; Li, J.; Lin, Y. Nitrogen-doped graphene and its application in electrochemical biosensing. ACS Nano 2010, 4, 1790-1798.

(21) Wang, H.; Maiyalagan, T.; Wang, X. Review on recent progress in nitrogen-doped graphene: synthesis, characterization, and its potential applications. ACS Catal. 2012, 2, 781-794.

(22) Cui, J.; Liang, S.; Zhang, J. A multifunctional material of twodimensional g-C4N3/graphene bilayer. Phys. Chem. Chem. Phys. 2016, $18,25388-25393$.

(23) Hashmi, A.; Farooq, M. U.; Hu, T.; Hong, J. Spin-Dependent Transport and Optical Properties of Transparent Half-Metallic g-C4N3 Films. J. Phys. Chem. C 2015, 119, 1859-1866.

(24) Li, X.; Zhang, S.; Wang, Q. Stability and physical properties of a tri-ring based porous g-C4N3 sheet. Phys. Chem. Chem. Phys. 2013, 15, $7142-7146$

(25) Bojdys, M. J.; Severin, N.; Rabe, J. P.; Cooper, A. I.; Thomas, A.; Antonietti, M. Exfoliation of crystalline 2D carbon nitride: thin sheets, scrolls and bundles via mechanical and chemical routes. Macromol. Rapid Commun. 2013, 34, 850-854.

(26) Becke, A. D. Density-functional thermochemistry. III. The role of exact exchange. J. Chem. Phys. 1993, 98, 5648-5652.

(27) Vosko, S. H.; Wilk, L.; Nusair, M. Accurate spin-dependent electron liquid correlation energies for local spin density calculations: a critical analysis. Can. J. Phys. 1980, 58, 1200-1211.

(28) Dovesi, R.; Saunders, V. R.; Roetti, C.; Orlando, R.; ZicovichWilson, C. M.; Pascale, F.; Civalleri, B.; Doll, K.; Harrison, N. M.; Bush, L. J.; et al. Crystal 14 User's Manual, University of Torino: Torino, Italy 2016.

(29) Dovesi, R.; Orlando, R.; Erba, A.; Zicovich-Wilson, C. M.; Civalleri, B.; Casassa, S.; Maschio, L.; Ferrabone, M.; De La Pierre, M.; D'Arco, P.; et al. CRYSTAL14: A program for theab initioinvestigation of crystalline solids. Int. J. Quantum Chem. 2014, 114, 1287-1317.

(30) Zicovich-Wilson, C. M.; Pascale, F.; Roetti, C.; Saunders, V. R.; Orlando, R.; Dovesi, R. Calculation of the vibration frequencies of ?-quartz: The effect of Hamiltonian and basis set. J. Comput. Chem. 2004, 25, 1873-1881.

(31) Pascale, F.; Zicovich-Wilson, C. M.; López Gejo, F.; Civalleri, B.; Orlando, R.; Dovesi, R. The Calculation of the vibrational frequencies of crystalline compounds and its implementation in the crystal code. J. Comput. Chem. 2004, 25, 888-897.

(32) Maschio, L.; Kirtman, B.; Rérat, M.; Orlando, R.; Dovesi, R. Ab initio analytical Raman intensities for periodic systems through a coupled perturbed Hartree-Fock/Kohn-Sham method in an atomic orbital basis. II. Validation and comparison with experiments. J. Chem. Phys. 2013, 139, 164102.

(33) Frisch, M. J.; Trucks, G. W.; Schlegel, H. B.; Scuseria, G. E.; Robb, M. A.; Cheeseman, J. R.; Scalmani, G.; Barone, V.; Petersson, G. A.; Nakatsuji, H.; et al. Gaussian 09, Revision A.02; Gaussian, Inc: Wallingford CT, 2009.

(34) Ladva, S. A.; Travis, W.; Quesada-Cabrera, R.; Rosillo-Lopez, M.; Afandi, A.; Li, Y.; Jackman, R. B.; Bear, J. C.; Parkin, I. P.; Blackman, C.; et al. Nanoscale, conformal films of graphitic carbon nitride deposited at room temperature: a method for construction of heterojunction devices. Nanoscale 2017, 9, 16586-16590.
(35) Chung, D. D. L. Review graphite. J. Mater. Sci. 2002, 37, 14751489.

(36) Dresselhaus, M. S.; Dresselhaus, G. Intercalation compounds of graphite. Adv. Phys. 2002, 51, 1-186.

(37) Crist, B. V. A review of XPS data-banks. XPS Rep 2007, 1, 1-52.

(38) Greczynski, G.; Hultman, L. C 1s Peak of Adventitious Carbon Aligns to the Vacuum Level: Dire Consequences for Material's Bonding Assignment by Photoelectron Spectroscopy. ChemPhysChem 2017, 18, $1507-1512$.

(39) Kwan, Y. C. G.; Ng, G. M.; Huan, C. H. A. Identification of functional groups and determination of carboxyl formation temperature in graphene oxide using the XPS O 1s Spectrum. Thin Solid Films 2015, $590,40-48$

(40) Fu, C. J.; Zhao, G. G.; Zhang, H. J.; Li, S. Evaluation and characterization of reduced graphene oxide nanosheets as anode materials for lithium-ion batteries. Int. J. Electrochem. Sci. 2013, 8, $6269-6280$

(41) Błoński, P.; Tuček, J.; Sofer, Z.; Mazánek, V.; Petr, M.; Pumera, M.; Otyepka, M.; Zbořil, R. Doping with graphitic nitrogen triggers ferromagnetism in graphene. J. Am. Chem. Soc. 2017, 139, 3171-3180.

(42) Dementjev, A. P.; de Graaf, A.; van de Sanden, M. C. M.; Maslakov, K. I.; Naumkin, A. V.; Serov, A. A. X-Ray photoelectron spectroscopy reference data for identification of the $\mathrm{C} 3 \mathrm{~N} 4$ phase in carbon-nitrogen films. Diamond Relat. Mater. 2000, 9, 1904-1907.

(43) Miller, T. S.; Jorge, A. B.; Suter, T. M.; Sella, A.; Corà, F.; McMillan, P. F. Carbon nitrides: synthesis and characterization of a new class of functional materials. Phys. Chem. Chem. Phys. 2017, 19, 1561315638.

(44) Luo, Z.; Lim, S.; Tian, Z.; Shang, J.; Lai, L.; MacDonald, B.; Fu, C.; Shen, Z.; Yu, T.; Lin, J. Pyridinic N doped graphene: synthesis, electronic structure, and electrocatalytic property. J.Mater. Chem. 2011, $21,8038-8044$

(45) Li, Z.; Lui, C. H.; Cappelluti, E.; Benfatto, L.; Mak, K. F.; Carr, G. L.; Shan, J.; Heinz, T. F. Structure-dependent fano resonances in the infrared spectra of phonons in few-layer graphene. Phys. Rev. Lett. 2012, 108, 156801.

(46) Rodil, S. E.; Ferrari, A. C.; Robertson, J.; Muhl, S. Infrared spectra of carbon nitride films. Thin Solid Films 2002, 420-421, 122-131.

(47) Cheng, Y. H.; Tay, B. K.; Lau, S. P.; Shi, X.; Qiao, X. L.; Chen, J. G.; Wu, Y. P.; Xie, C. S. Raman spectroscopy of carbon nitride films deposited using the filtered cathodic vacuum-arc technique combined with a radio-frequency nitrogen-ion beam. Appl. Phys. A 2001, 73, 341345.

(48) Ferrari, A. C.; Rodil, S. E.; Robertson, J. Resonant Raman spectra of amorphous carbon nitrides: the G peak dispersion. Diamond Relat. Mater. 2003, 12, 905-910.

(49) Casiraghi, C.; Ferrari, A. C.; Robertson, J. Raman Spectroscopy of hydrogenated amorphous carbons. Phys. Rev. B 2005, 72, 085401.

(50) Ferrari, A. C.; Rodil, S. E.; Robertson, J. Interpretation of infrared and Raman spectra of amorphous carbon nitrides. Phys. Rev. B 2003, 67, 155306.

(51) Ferrari, A. C. Raman spectroscopy of graphene and graphite: disorder, electron-phonon coupling, doping and nonadiabatic effects. Solid State Commun. 2007, 143, 47-57.

(52) Ferrari, A. C.; Basko, D. M. Raman spectroscopy as a versatile tool for studying the properties of graphene. Nat. Nanotechnol. 2013, 8, 235-246.

(53) Rodil, S. E.; Ferrari, A. C.; Robertson, J.; Milne, W. I. Raman and infrared modes of hydrogenated amorphous carbon nitride. J. Appl. Phys. 2001, 89, 5425-5430.

(54) Kay, C. W. M.; Schleicher, E.; Hitomi, K.; Todo, T.; Bittl, R.; Weber, S. Determination of the G-matrix orientation in flavin radicals by high-field/high-frequency electron-nuclear double resonance. Magn. Reson. Chem. 2005, 43, S96-S102.

(55) Lau, V. W.-h.; Klose, D.; Kasap, H.; Podjaski, F.; Pignié, M.-C.; Reisner, E.; Jeschke, G.; Lotsch, B. V. Dark photocatalysis: storage of solar energy in carbon nitride for time-delayed hydrogen generation. Angew. Chem., Int. Ed. 2017, 56, 510-514. 
(56) Kuzuhara, D.; Yamada, H.; Xue, Z. L.; Okujima, T.; Mori, S.; Shen, Z.; Uno, H. New synthesis of meso-free-[14] triphyrin(2.1.1) by Mcmurry coupling and its derivatization to $\mathrm{Mn}(\mathrm{I})$ and $\operatorname{Re}(\mathrm{I})$ complexes. Chem. Commun. 2011, 47, 722-724. 


\section{Synthesis, Structure and Electronic Properties of Graphitic Carbon Nitride Films}

Theo Suter*†o, Veronika Brázdová $\uparrow$, Kit McColl $\uparrow$, Thomas S. Miller†o, Hiroki Nagashima $\ddagger \S \nabla$, Enrico

Salvadorił $\mathbf{\Omega}$, Andrea Sella†, Christopher A. Howard\|, Christopher W. M. Kay $\downarrow$, Furio Corà $†$, Paul F. McMillan*†

*Corresponding authors

$\dagger$ Department of Chemistry, Christopher Ingold Building, University College London, 20 Gordon Street, London WC1H

0AJ, United Kingdom

I| Department of Physics and Astronomy, University College London, Gower Street, London WC1E 6BT, U.K.

¥Institute of Structural and Molecular Biology and London Centre for Nanotechnology, University College London WE1E 6BT, UK

$\Omega$ School of Biological and Chemical Sciences, Queen Mary University of London, London E1 4NS, UK

$\S$ Division of Material Science, Graduate School of Science, Nagoya University, Furo-cho, Chikusa, Nagoya, 464-8602, Japan

$\perp$ Department of Chemistry, University of Saarland, 66123 Saarbrücken, Germany.

$\nabla$ Molecular Photoscience Research Center, Kobe University, 1-1 Rokkodai-cho, Nada-ku, Kobe 657-8501, Japan.

${ }^{\mathrm{O}}$ Electrochemical Innovation Lab, University College London, London WC1E 7JE, United Kingdon 


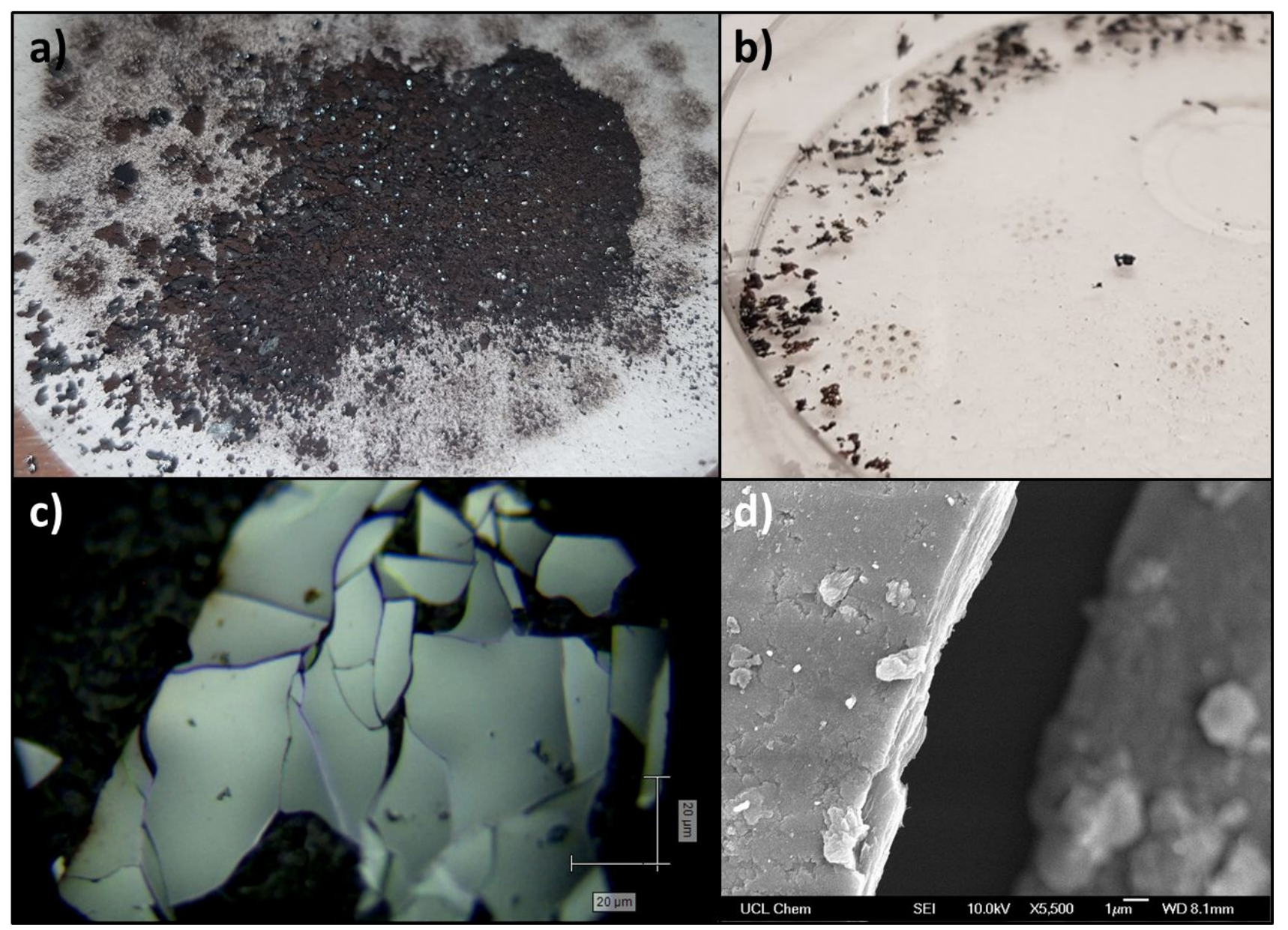

Figure S1. Images of the graphitic layered carbon nitride materials recovered following synthesis. a) Photograph of recovered bulk material showing the dark brown powder mixed with small black reflective flakes. b) The larger black reflective flakes were separated by hand from the brown powder mixture. c) Optical image showing the highly reflective near-planar surfaces of the cleaved flakes. The scale bar indicates $20 \mathrm{~mm}$. d) SEM image (scale bar $1 \mathrm{~mm}$ ) of the edge structure of a flake, showing evidence for the layered stacking. 

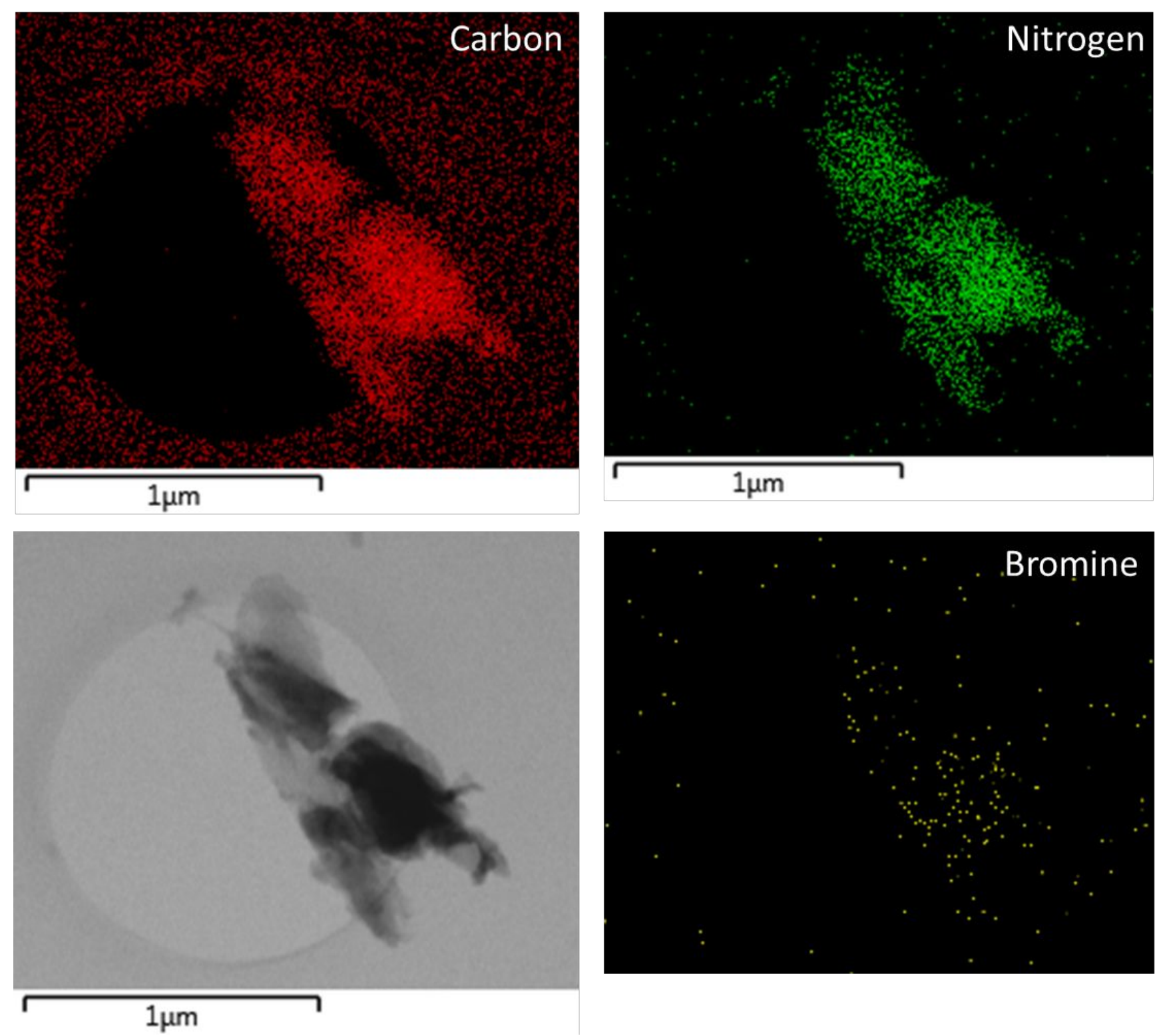

Figure S2. Energy dispersive X-ray analysis and mapping results obtained in low resolution TEM studies of carbon nitride flakes showing atomic distributions within the samples. The sparsely distributed spots for $\mathrm{Br}$ arise from randomly dispersed $\mathrm{KBr} / \mathrm{LiBr}$ nanocrystals re-deposited on the sample surface derived from the alkali halide reaction medium after washing in water. The $\mathrm{C}$ and $\mathrm{N}$ 
concentrations closely match each other indicating a homogeneous distribution of these elements within the samples.
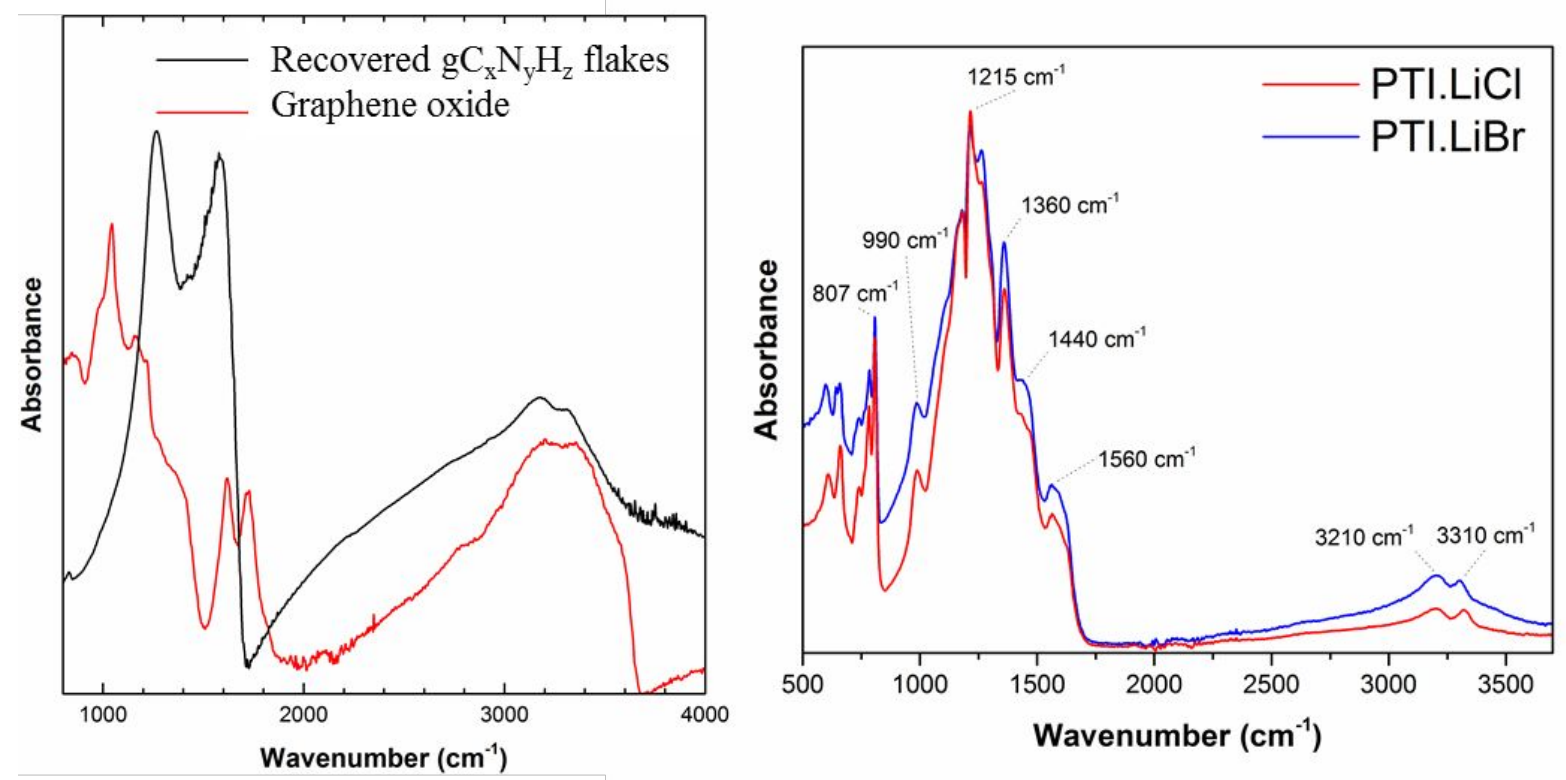

Figure S3. a) IR spectra of recovered carbon nitride flakes compared with graphene oxide (GO). b) IR spectra of PTI.LiCl and PTI.LiBr crystalline compounds. 


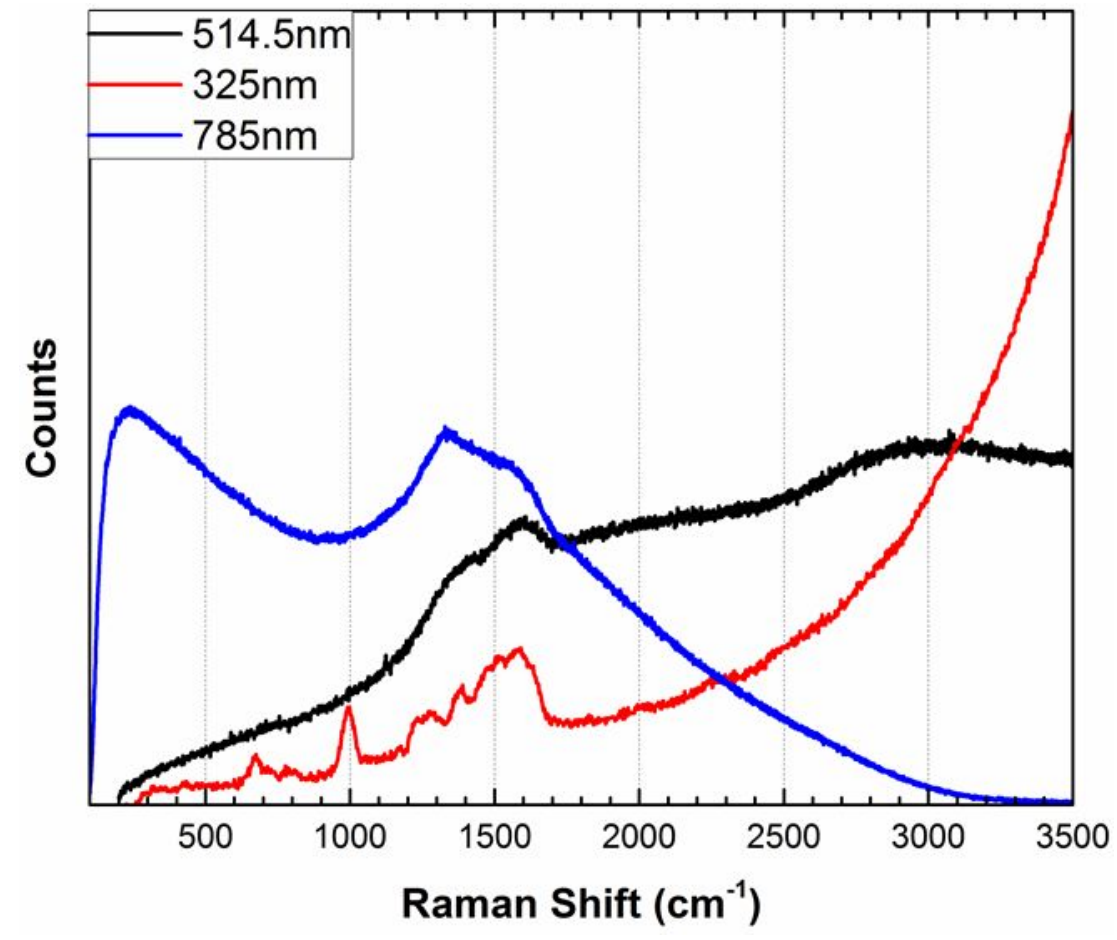

Figure S4. Raman spectra of crystalline PTI.LiBr using different excitation wavelengths $(325,514.5$ and $785 \mathrm{~nm})$.
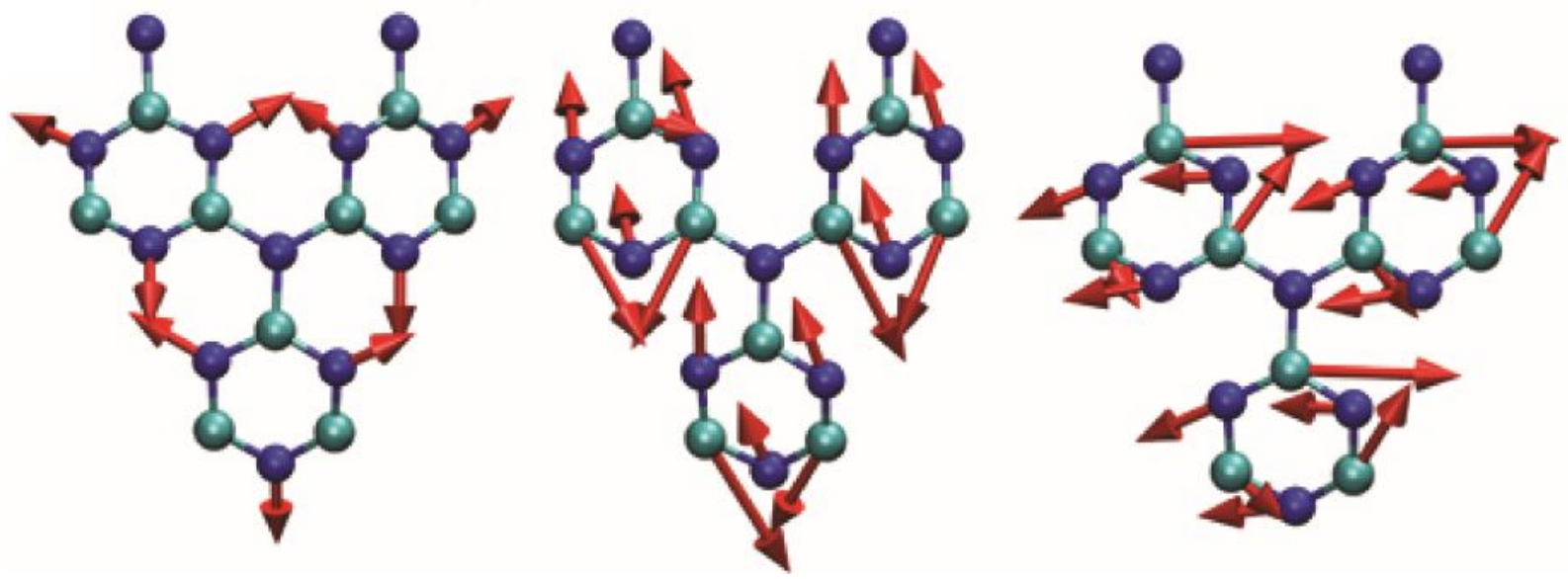

Figure S5. Calculated (DFT) atomic displacement patterns for a single layer of triazine-based g- $\mathrm{C}_{3} \mathrm{~N}_{4}$. At left we show the strongly Raman active mode expected to occur near $1000 \mathrm{~cm}^{-1}$. The other two 
modes correspond to in-phase Raman-active breathing motions that predicted to occur near 1044 and $1550 \mathrm{~cm}^{-1}$. None of these modes were observed experimentally.
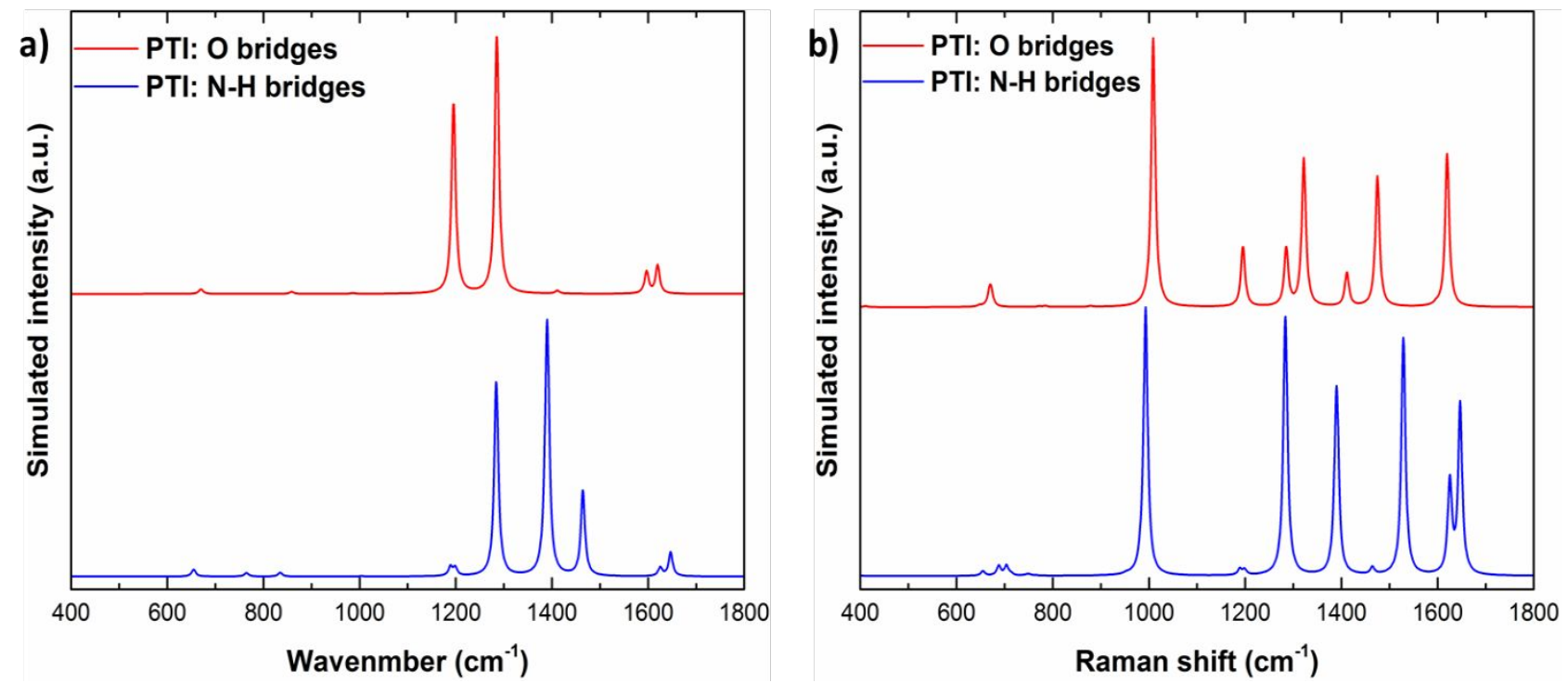

Figure S6. DFT simulated vibrational spectra of intercalant-free PTI $\left(\mathrm{C}_{6} \mathrm{~N}_{9} \mathrm{H}_{3}\right)$ and oxygen bridged PTI layered structures. a) Comparison of IR spectra for both structures. b) Comparison of simulated Raman spectra for both structures. Both models predict strongly Raman active peaks in the $\sim 1000 \mathrm{~cm}-1$ region.

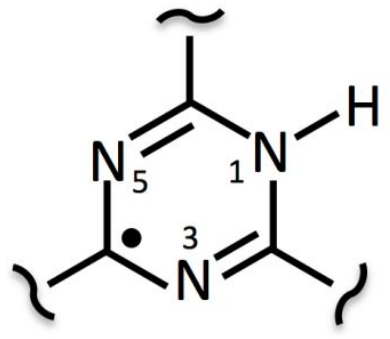

Scheme S1. Molecular structure of the core of the putative radical in TGCN with the molecular numbering scheme.

The $3 \mathrm{~N}$ atoms and the proton are expected to have hfcs, as summarized in Table S1. 


\begin{tabular}{|c|c|c|c|c|}
\hline Position & $\mathrm{A}_{\text {iso }}$ & $\mathrm{T}_{\mathrm{x}}$ & $\mathrm{T}_{\mathrm{y}}$ & $\mathrm{T}_{\mathrm{z}}$ \\
\hline $\mathrm{N}(1)$ & 15.8 & -6.4 & -6.4 & 12.9 \\
\hline $\mathrm{N}(1) \mathrm{H}$ & 33.8 & -6.6 & 12.2 & -5.6 \\
\hline $\mathrm{N}(3)$ & -4.2 & 3.1 & 3.8 & -6.9 \\
\hline $\mathrm{N}(5)$ & -3.5 & 2.7 & 3.4 & -6.1 \\
\hline
\end{tabular}

Table S1. Isotropic and anisotropic hfe tensor components. All values are given in $\mathrm{MHz}$. All $3 \mathrm{~N}$ atoms have axially symmetric dipolar tensor components, with the Tz component which is perpendicular to the molecular plane being the largest. The largest spin density is on $\mathrm{N}(1)$, whilst $\mathrm{N}(3)$ and $\mathrm{N}(5)$ which are chemically equivalent, have similar and smaller hfcs. $\mathrm{N}(1) \mathrm{H}$, however, also has a large hfc, but in this case, the largest component is in the plane, and perpendicular to the $\mathrm{N}-\mathrm{H}$ bond. 
a)

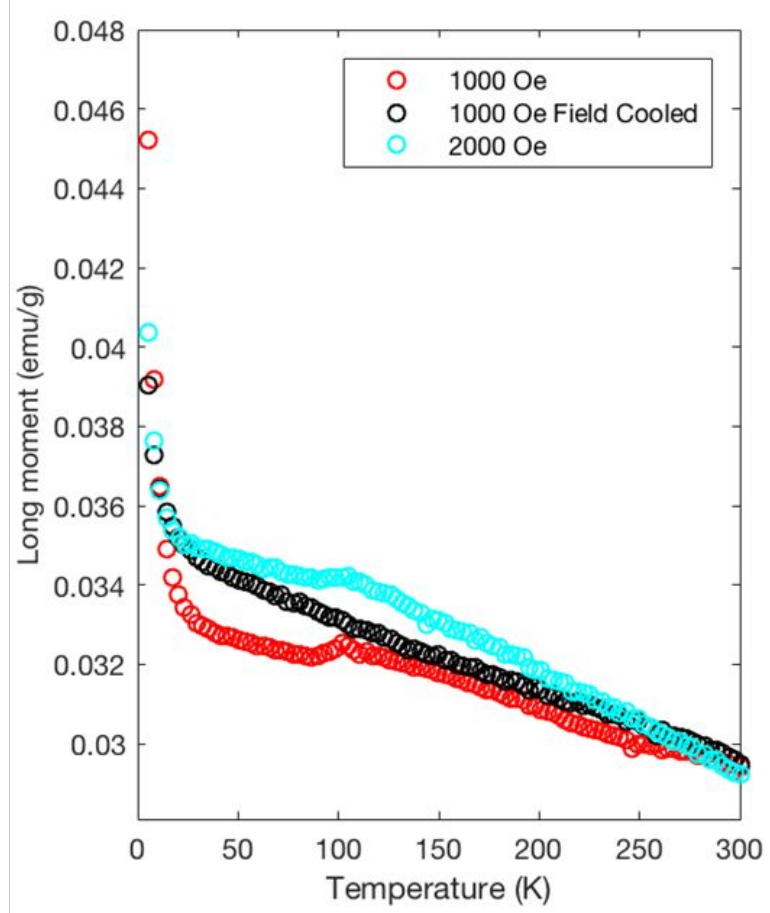

b)

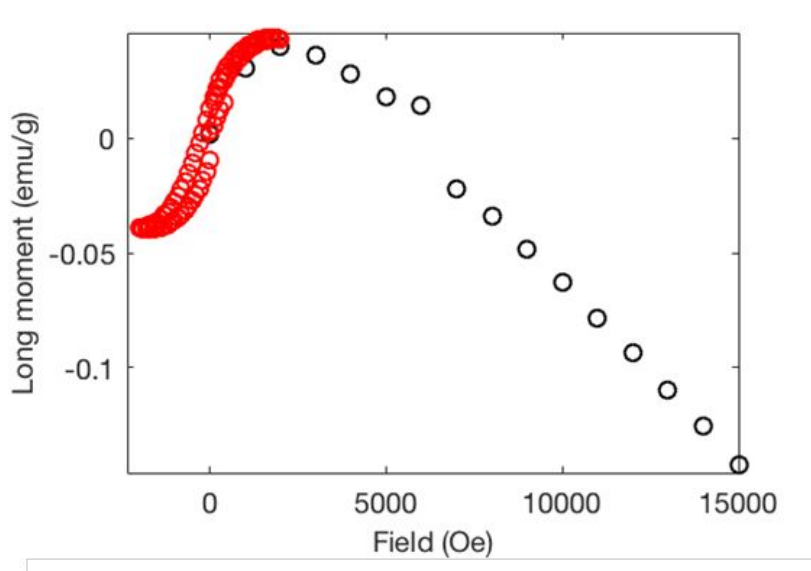

Figure S7. a) Magnetization recorded as a function of temperature upon warming showing data for: a) a zero field cooled sample measured in 1000 Oe (red circles) and 2000 Oe (cyan circles) applied field, and the same sample measured upon warming after having been cooled in a 1000 Oe field (black circles). The 5-300 K scans are generally consistent with a paramagnetic response and potentially antiferromagnetic ordering. The data display a small transition near $100 \mathrm{~K}$, that disappears after cooling the sample in a magnetic field and then studied during re-warming. b) Magnetization recorded as a function of field strength at $5 \mathrm{~K}$. The data show evidence for a paramagnetic response with a moment that saturated at $\sim 200 \mathrm{mT}$. At higher field, the sample exhibited a diamagnetic response revealing additional complexity to the magnetic properties that will require further investigation. 